\title{
Eriophyoid mites (Acari: Eriophyoidea) associated with palm trees
}

\author{
DENISE NAVIA ${ }^{1}$, MANOEL G. C. GONDIM JUNIOR ${ }^{2} \&$ GILBERTO J. DE MORAES ${ }^{3}$

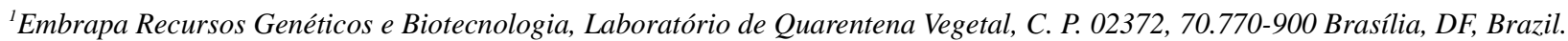 \\ E-mail:navia@cenargen.embrapa.br \\ ${ }^{2}$ Universidade Federal Rural de Pernambuco, Departamento de Agronomia, 51171-900 Recife, PE, Brazil. \\ E-mail: mguedes@ufrpe.br \\ ${ }^{3}$ CNPq-Brazil Researcher, Universidade de São Paulo/ESALQ, Departamento de Entomologia, Fitopatologia e Zoologia Agrícola, \\ 13.418-900 Piracicaba, SP, Brazil.E-mail: gjmoraes@carpa.ciagri.usp.br
}

\section{Table of contents}

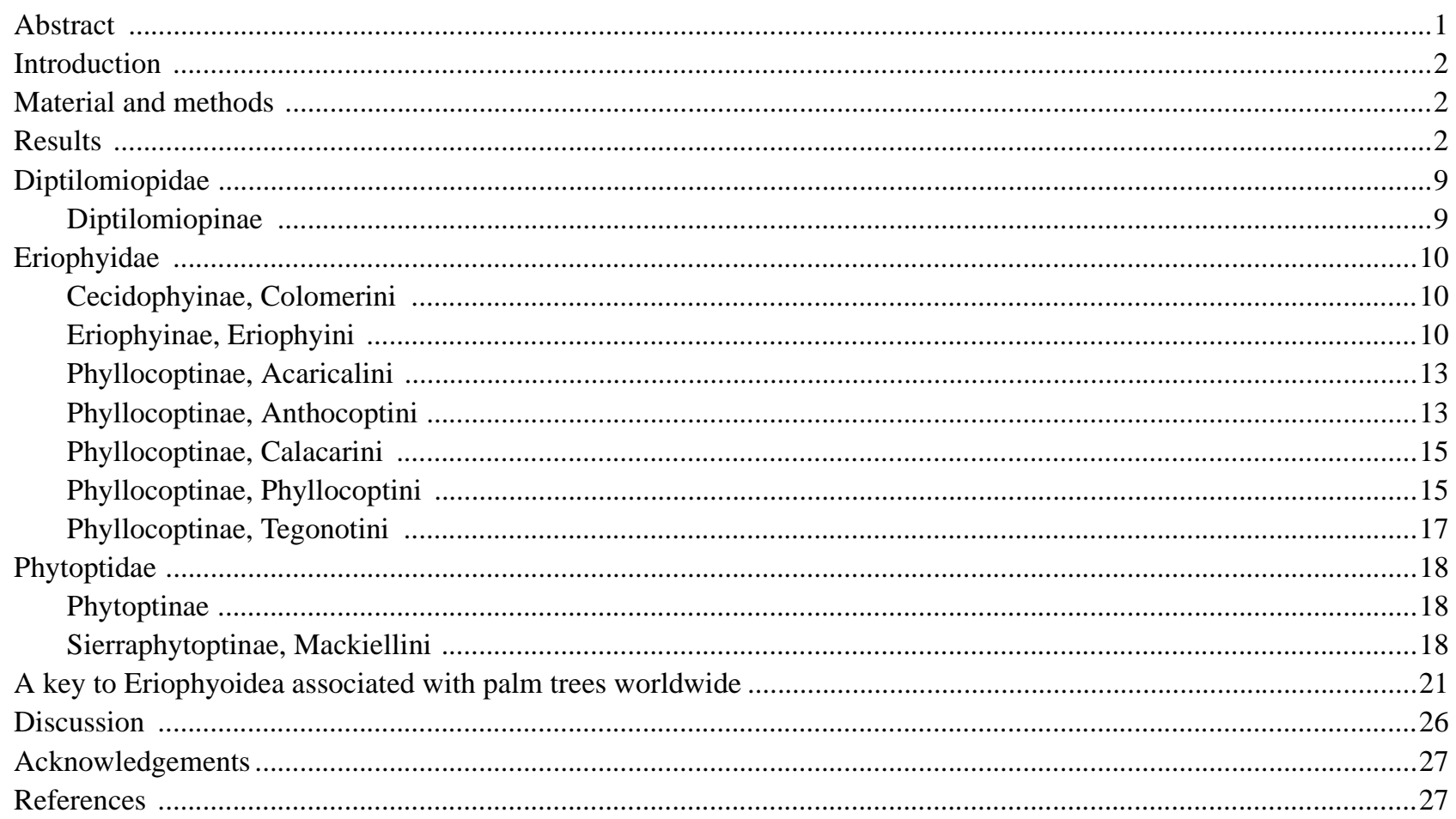

\begin{abstract}
Information is presented on eriophyoid mites found on palm trees worldwide by different authors, including original data from a recent survey conducted in Brazil, Costa Rica and Mexico. For each species, information on synonymy, locations where it was found on palm trees, palm hosts, and damage are included. Sixty-two eriophyoid species from 31 genera, associated with 54 palm tree species from 25 genera, are listed. A dichotomous key is provided to help in the separation of the reported mites. Four eriophyoid species are reported on palm trees in Europe; 6 in Africa; 17 in Asia, Pacific Islands and Australia; and 40 in the Americas. Four of the reported species belong to Diptilomiopidae, 44 to Eriophyidae and 14 to Phytoptidae. The need for further studies on these mites around the world is discussed.
\end{abstract}

Key words: Prostigmata, Arecaceae, taxonomy, Eriophyidae, Diptilomiopidae, Phytoptidae, phytophagous mites 


\section{Introduction}

Palm trees are monocotyledons of the family Arecaceae (before Palmaceae or Palmae). They constitute a highly diverse group, presently composed of about 3,500 species of 240 genera, found especially in tropical and subtropical areas (Lorenzi 1996). Some of these plants are of extreme socio-economic importance. Areas in which several palm species are cultivated, especially coconuts and oil palms, have increased substantially in the last decade, especially in Brazil.

Eriophyoidea mites (Acari: Prostigmata) are strictly phytophagous and can reach pest status in agricultural systems, being considered the second most economically important group of mite pests (Lindquist et al. 1996).

Information on eriophyoids associated with palm trees worldwide is scattered. Several papers with new reports or descriptions of new taxa on palms have been published in the last 20 years (for ex. in Flechtmann \& Santana 1997; Flechtmann 1989, 1994, 1998; Gondim et al. 2000; Navia \& Flechtmann 2002, 2003a; Santana \& Flechtmann 1998; Santana et al. 1994), however many papers on the subject were published long ago and are difficult to access. This paper synthesizes the main information published on host plants, damage and geographic distribution, adds original information concerning new host plants, locality or infested plant part of eriophyoids on palm trees and provides a dichotomous key to the mite species reported.

\section{Material and methods}

Retrieval of published information was largely based on Amrine Jr. \& Stasny (1994) and on the database developed by Amrine Jr. \& de Lillo (2003). Information about species of economic importance (for ex. Aceria guerreronis Keifer, 1965 and Retracrus elaeis Keifer 1975a) was restricted to the first report in the country, on the host plant or on the plant part, considering that it would not be viable in this paper to refer to the extensive literature on damage, control and natural enemies of these species.

Original data refer to non-systematic sampling conducted from 1997 to 2003. Plant parts of nearly 200 species of native or introduced palm trees were collected in the States of Amazonas, Pernambuco, Rio de Janeiro and São Paulo, Brazil, as well as in the States of Quintana Roo and Yucatan, Mexico, and Costa Rica. These parts were taken to the laboratory and examined for the presence of eriophyoid mites. The mites were mounted in Berlese modified medium (Amrine Jr. \& Manson 1996) for subsequent identification. Specimens collected during these surveys were deposited in the collection of "Departamento de Entomologia, Fitopatologia e Zoologia Agrícola, Universidade de São Paulo, ESALQ", Piracicaba, São Paulo, Brazil and of "Laboratório de Quarentena Vegetal, Embrapa Recursos Genéticos e Biotecnologia", Brasilia, DF, Brazil.

A dichotomous key to known eriophyoid mite species associated with palm trees was prepared, based initially on the key to genera of Amrine Jr. et al. (2003), complemented by characteristics from the original description of each species.

\section{Results}

Sixty-two eriophyoid species of 31 genera have been found on 54 palm tree species of 25 genera around the world. Each of these palm species can host several eriophyoid species. Some species were restricted to particular plant parts, whereas others were found on various plant parts.

The present survey conducted from 1997 to 2003 in Brazil, Mexico and Costa Rica resulted in 31 new host plant records for 10 Eriophyoidea species; new part of plant infested for four species and new country records for two species. Collection data of the present survey are presented in Table 1. 
TABLE 1. Eriophyoidea associated with native and introduced palms in Brazil, Costa Rica and Mexico, including new collecting records. (Infested plant parts (PP): L — leaves; TS — terminal shoot; F — fruits) (States of Brazil: AM — Amazonas; PE — Pernambuco; RJ — Rio de Janeiro; SP — São Paulo) ( ${ }^{1}$ new host plant record; ${ }^{2}$ new plant part infested; ${ }^{3}$ new country record).

\begin{tabular}{|c|c|c|c|c|c|c|}
\hline Eriophyoidea Mite & Palm Host & $\mathrm{PP}$ & Locality & Lat/Long & Date & Collector \\
\hline \multirow[t]{6}{*}{ Amrineus cocofolius } & Attalea geraensis ${ }^{1}$ & $\mathrm{~L}$ & $\begin{array}{l}\text { São Carlos, SP, } \\
\text { Brazil }\end{array}$ & $\begin{array}{l}21^{\circ} 54^{\prime} 50^{\prime \prime} \mathrm{S} ; \\
47^{\circ} 49^{\prime} 21^{\prime \prime} \mathrm{W}\end{array}$ & XII-2000 & D. Navia \\
\hline & Butia archeri ${ }^{1}$ & $\mathrm{~L}$ & $\begin{array}{l}\text { Piracicaba, SP, } \\
\text { Brazil }\end{array}$ & $\begin{array}{l}22^{\circ} 42^{\prime} 30^{\prime \prime} \mathrm{S}, \\
47^{\circ} 38^{\prime} 00^{\prime \prime} \mathrm{W}\end{array}$ & V-2001 & D. Navia \\
\hline & Butia eriospatha ${ }^{1}$ & $\mathrm{~L}$ & $\begin{array}{l}\text { Piracicaba, SP, } \\
\text { Brazil }\end{array}$ & $\begin{array}{l}22^{\circ} 42^{\prime} 30^{\prime \prime} \mathrm{S}, \\
47^{\circ} 38^{\prime} 00 ” \mathrm{~W}\end{array}$ & $X-2000$ & D. Navia \\
\hline & Cocos nucifera & $\mathrm{L}$ & $\begin{array}{l}\text { Piracicaba, SP, } \\
\text { Brazil }\end{array}$ & $\begin{array}{l}22^{\circ} 42^{\prime} 30^{\prime \prime} \mathrm{S} ; \\
47^{\circ} 48^{\prime} 00^{\prime \prime} \mathrm{W}\end{array}$ & $\begin{array}{l}\text { III, VIII- } \\
1998\end{array}$ & M. G. C. Gondim Jr. \\
\hline & Cocos nucifera & $\mathrm{L}$ & $\begin{array}{l}\text { Recife, SP, } \\
\text { Brazil }\end{array}$ & $\begin{array}{l}08^{\circ} 10^{\prime} 52^{\prime \prime} \mathrm{S} ; \\
34^{\circ} 54^{\prime} 47^{\prime \prime} \mathrm{W}\end{array}$ & $\begin{array}{l}\text { IV-1998, I- } \\
1999\end{array}$ & M. G. C. Gondim Jr. \\
\hline & Syagrus romanzoffiana $^{1}$ & $\mathrm{~F}$ & $\begin{array}{l}\text { Piracicaba, SP, } \\
\text { Brazil }\end{array}$ & $\begin{array}{l}22^{\circ} 42^{\prime} 30^{\prime \prime} \mathrm{S}, \\
47^{\circ} 38^{\prime} 00^{\prime \prime} \mathrm{W}\end{array}$ & III-2000 & D. Navia \\
\hline \multirow[t]{4}{*}{ Davisella palmea } & Euterpe edulis $^{1}$ & $\mathrm{~L}$ & $\begin{array}{l}\text { Cananéia, SP, } \\
\text { Brazil }\end{array}$ & $\begin{array}{l}24^{\circ} 53^{\prime} 45^{\prime \prime} \mathrm{S} \\
47^{\circ} 50^{\prime} 17^{\prime \prime} \mathrm{W}\end{array}$ & IV, X-1998 & M. G. C. Gondim Jr. \\
\hline & & $\mathrm{L}$ & $\begin{array}{l}\text { Pariquera-Açu, } \\
\text { SP, Brazil }\end{array}$ & $\begin{array}{l}24^{\circ} 36^{\prime} 41^{\prime \prime} \mathrm{S} ; \\
47^{\circ} 53^{\prime} 23^{\prime \prime} \mathrm{W}\end{array}$ & IV, X-1998 & M. G. C. Gondim Jr. \\
\hline & Syagrus romanzoffiana & $\mathrm{L}$ & $\begin{array}{l}\text { Cananéia, SP, } \\
\text { Brazil }\end{array}$ & $\begin{array}{l}24^{\circ} 53^{\prime} 45^{\prime \prime} \mathrm{S} ; \\
47^{\circ} 50^{\prime} 17^{\prime \prime} \mathrm{W}\end{array}$ & IV, X-1998 & M. G. C. Gondim Jr. \\
\hline & & $\mathrm{L}$ & $\begin{array}{l}\text { Pariquera-Açu, } \\
\text { SP, Brazil }\end{array}$ & $\begin{array}{l}24^{\circ} 36^{\prime} 41^{\prime \prime} \mathrm{S} ; \\
47^{\circ} 53^{\prime} 23^{\prime \prime} \mathrm{W}\end{array}$ & IV, X-1998 & M. G. C. Gondim Jr. \\
\hline Knorella geonomae & Geonoma schottiana & $\begin{array}{l}\mathrm{TS} \\
2\end{array}$ & $\begin{array}{l}\text { Cananéia, SP, } \\
\text { Brazil }\end{array}$ & $\begin{array}{l}24^{\circ} 53^{\prime} 45^{\prime \prime} \mathrm{S} ; \\
47^{\circ} 50^{\prime} 17^{\prime \prime} \mathrm{W}\end{array}$ & VIII-2000 & D. Navia \\
\hline \multirow[t]{2}{*}{ Notostrix attenuata } & Acrocomia aculeata $^{I}$ & $\mathrm{~L}$ & $\begin{array}{l}\text { Piracicaba, SP, } \\
\text { Brazil }\end{array}$ & $\begin{array}{l}22^{\circ} 42^{\prime} 30^{\prime \prime} \mathrm{S} ; \\
47^{\circ} 48^{\prime} 00^{\prime \prime} \mathrm{W}\end{array}$ & $\begin{array}{l}\text { III, VIII- } \\
1998\end{array}$ & M. G. C. Gondim Jr. \\
\hline & Syagrus romanzoffiana ${ }^{1}$ & $\mathrm{~L}$ & $\begin{array}{l}\text { Piracicaba, SP, } \\
\text { Brazil }\end{array}$ & $\begin{array}{l}22^{\circ} 42^{\prime} 30^{\prime \prime} \mathrm{S} ; \\
47^{\circ} 48^{\prime} 00^{\prime \prime} \mathrm{W}\end{array}$ & $\begin{array}{l}\text { III, VIII- } \\
1998\end{array}$ & M. G. C. Gondim Jr. \\
\hline \multirow[t]{7}{*}{ Notostrix butiae } & Attalea geraensis ${ }^{1}$ & $\mathrm{~L}$ & $\begin{array}{l}\text { Luiz Antonio, } \\
\text { SP, Brazil }\end{array}$ & $\begin{array}{l}21^{\circ} 36^{\prime} 13^{\prime \prime} \mathrm{S} ; \\
47^{\circ} 47^{\prime} 17^{\prime \prime} \mathrm{W}\end{array}$ & XII-2000 & D. Navia \\
\hline & & $\mathrm{L}$ & $\begin{array}{l}\text { São Carlos, SP, } \\
\text { Brazil }\end{array}$ & $\begin{array}{l}21^{\circ} 54^{\prime} 50^{\prime \prime} \mathrm{S} \\
47^{\circ} 49^{\prime} 21^{\prime \prime} \mathrm{W}\end{array}$ & XII-2000 & D. Navia \\
\hline & Butia archeri ${ }^{1}$ & $\mathrm{~L}$ & $\begin{array}{l}\text { Pirassununga, } \\
\text { SP, Brazil }\end{array}$ & $\begin{array}{l}21^{\circ} 56^{\prime} 20^{\prime \prime} \mathrm{S} \\
47^{\circ} 28^{\prime} 26^{\prime \prime} \mathrm{W}\end{array}$ & $\begin{array}{l}\text { VII, XII- } \\
2000\end{array}$ & D. Navia \\
\hline & & $\begin{array}{l}\mathrm{TS} \\
2\end{array}$ & $\begin{array}{l}\text { Pirassununga, } \\
\text { SP, Brazil }\end{array}$ & $\begin{array}{l}21^{\circ} 56^{\prime} 20^{\prime \prime} \mathrm{s} ; \\
47^{\circ} 28^{\prime} 26^{\prime \prime} \mathrm{W}\end{array}$ & $\begin{array}{l}\text { VII, XII- } \\
2000\end{array}$ & D. Navia \\
\hline & 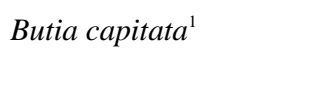 & $\mathrm{L}$ & $\begin{array}{l}\text { Campinas, SP, } \\
\text { Brazil }\end{array}$ & $\begin{array}{l}24^{\circ} 36^{\prime} 41^{\prime \prime} \mathrm{S}, \\
47^{\circ} 53^{\prime} 23^{\prime \prime} \mathrm{W}\end{array}$ & VI-2000 & D. Navia \\
\hline & Syagrus microphylla ${ }^{1}$ & $\mathrm{~L}$ & $\begin{array}{l}\text { Pirassununga, } \\
\text { SP, Brazil }\end{array}$ & $\begin{array}{l}21^{\circ} 56^{\prime} 20^{\prime \prime} \mathrm{S} \\
47^{\circ} 28^{\prime} 26^{\prime \prime} \mathrm{W}\end{array}$ & $\begin{array}{l}\text { VII, XI- } \\
2000\end{array}$ & D. Navia \\
\hline & Syagrus quinquefaria ${ }^{l}$ & $\mathrm{~L}$ & $\begin{array}{l}\text { Piracicaba, SP, } \\
\text { Brazil }\end{array}$ & $\begin{array}{l}22^{\circ} 42^{\prime} 30^{\prime \prime} \mathrm{S}, \\
47^{\circ} 38^{\prime} 00^{\prime \prime} \mathrm{W}\end{array}$ & $X-2000$ & D. Navia \\
\hline \multirow[t]{2}{*}{ Notostrix exigua } & Euterpe edulis & $\mathrm{L}$ & $\begin{array}{l}\text { Cananéia, SP, } \\
\text { Brazil }\end{array}$ & $\begin{array}{l}24^{\circ} 53^{\prime} 45^{\prime \prime} \mathrm{S} ; \\
47^{\circ} 50^{\prime} 17^{\prime \prime} \mathrm{W}\end{array}$ & IV, X-1998 & M. G. C. Gondim Jr. \\
\hline & & $\mathrm{L}$ & $\begin{array}{l}\text { Pariquera-Açu, } \\
\text { SP, Brazil }\end{array}$ & $\begin{array}{l}24^{\circ} 36^{\prime} 41^{\prime \prime} \mathrm{S} ; \\
47^{\circ} 53^{\prime} 23^{\prime \prime} \mathrm{W}\end{array}$ & IV, X-1998 & M. G. C. Gondim Jr. \\
\hline
\end{tabular}




\begin{tabular}{|c|c|c|c|c|c|c|}
\hline & & $\mathrm{L}$ & $\begin{array}{l}\text { Piracicaba, SP, } \\
\text { Brazil }\end{array}$ & $\begin{array}{l}22^{\circ} 42^{\prime} 30^{\prime \prime} \mathrm{S}, \\
47^{\circ} 38^{\prime} 00^{\prime \prime} \mathrm{W}\end{array}$ & $X-2000$ & D. Navia \\
\hline & & $\mathrm{L}$ & $\begin{array}{l}\text { Teresópolis, } \\
\text { RJ, Brazil }\end{array}$ & $\begin{array}{l}22^{\circ} 29^{\prime} 23^{\prime \prime} \mathrm{S} \\
42^{\circ} 54^{\prime} 47^{\prime \prime} \mathrm{W}\end{array}$ & III-2001 & D. Navia \\
\hline & Bactris setosa ${ }^{1}$ & $\begin{array}{l}\mathrm{L}, \\
\mathrm{TS} \\
2\end{array}$ & $\begin{array}{l}\text { Pariquera-Açu, } \\
\text { SP, Brazil }\end{array}$ & $\begin{array}{l}24^{\circ} 36^{\prime} 41^{\prime \prime} \mathrm{S} \\
47^{\circ} 53^{\prime} 23^{\prime \prime} \mathrm{W}\end{array}$ & IV-2000 & D. Navia \\
\hline & Euterpe sp. & $\mathrm{L}$ & $\begin{array}{l}\text { Igarassu, PE, } \\
\text { Brazil }\end{array}$ & $\begin{array}{l}27^{\circ} 53^{\prime} 28^{\prime \prime} \mathrm{S} ; \\
34^{\circ} 58^{\prime} 35^{\prime \prime} \mathrm{W}\end{array}$ & $\begin{array}{l}\text { VI-1998; I- } \\
1999\end{array}$ & M. G. C. Gondim Jr. \\
\hline Notostrix nasutiformes & Cocos nucifera & $\mathrm{L}$ & $\begin{array}{l}\text { Chetumal, } \\
\text { Quintana Roo, } \\
\text { México }^{3}\end{array}$ & $\begin{array}{l}18^{\circ} 30,4 \mathrm{~N} \\
88^{\circ} 19,7 \mathrm{~W}\end{array}$ & IX-2002 & D. Navia \\
\hline \multirow[t]{3}{*}{ Propilus gentyi } & Astrocaryum aculeatissimum ${ }^{l}$ & $\mathrm{~L}$ & $\begin{array}{l}\text { Cananéia, SP, } \\
\text { Brazil }\end{array}$ & $\begin{array}{l}24^{\circ} 53^{\prime} 45^{\prime \prime} \mathrm{S} \\
47^{\circ} 50^{\prime} 17^{\prime \prime} \mathrm{W}\end{array}$ & IV, X-1998 & M. G. C. Gondim Jr. \\
\hline & & $\mathrm{L}$ & $\begin{array}{l}\text { Pariquera-Açu, } \\
\text { SP, Brazil }\end{array}$ & $\begin{array}{l}24^{\circ} 36^{\prime} 41^{\prime \prime} \mathrm{S} \\
47^{\circ} 53^{\prime} 23^{\prime \prime} \mathrm{W}\end{array}$ & IV, X-1998 & M. G. C. Gondim Jr. \\
\hline & Bactris vulgaris ${ }^{1}$ & $\mathrm{~L}$ & $\begin{array}{l}\text { Cananéia, SP, } \\
\text { Brazil }\end{array}$ & $\begin{array}{l}24^{\circ} 53^{\prime} 45^{\prime \prime} \mathrm{S} \\
47^{\circ} 50^{\prime} 17^{\prime \prime} \mathrm{W}\end{array}$ & $\mathrm{I}-2001$ & D. Navia \\
\hline \multirow[t]{4}{*}{ Propilus spinosus } & Bactris setosa ${ }^{1}$ & $\mathrm{~L}$ & $\begin{array}{l}\text { Pariquera-Açu, } \\
\text { SP, Brazil }\end{array}$ & $\begin{array}{l}24^{\circ} 36^{\prime} 41^{\prime \prime} \mathrm{S} \\
47^{\circ} 53^{\prime} 23^{\prime \prime} \mathrm{W}\end{array}$ & IV, X-1998 & M. G. C. Gondim Jr. \\
\hline & & $\mathrm{L}$ & $\begin{array}{l}\text { Jundiaí, SP, } \\
\text { Brazil }\end{array}$ & $\begin{array}{l}23^{\circ} 13^{\prime} 18^{\prime \prime} \mathrm{S} ; \\
46^{\circ} 55^{\prime} 16^{\prime \prime} \mathrm{W}\end{array}$ & $\begin{array}{l}\text { IX-2000; I- } \\
2001\end{array}$ & D. Navia \\
\hline & & $\mathrm{L}$ & $\begin{array}{l}\text { Pariquera-Açu, } \\
\text { SP, Brazil }\end{array}$ & $\begin{array}{l}24^{\circ} 36^{\prime} 41^{\prime \prime} \mathrm{S} \\
47^{\circ} 53^{\prime} 23^{\prime \prime} \mathrm{W}\end{array}$ & I, X-2000 & D. Navia \\
\hline & Syagrus romanzoffiana ${ }^{1}$ & $\mathrm{~L}$ & $\begin{array}{l}\text { Pariquera-Açu, } \\
\text { SP, Brazil }\end{array}$ & $\begin{array}{l}24^{\circ} 36^{\prime} 41^{\prime \prime} \mathrm{S} \\
47^{\circ} 53^{\prime} 23^{\prime \prime} \mathrm{W}\end{array}$ & X-1998 & M. G. C. Gondim Jr. \\
\hline \multirow[t]{4}{*}{ Propilus syagris } & Astrocaryum aculeatissimum ${ }^{1}$ & $\begin{array}{l}\mathrm{L}, \\
\mathrm{TS} \\
2\end{array}$ & $\begin{array}{l}\text { Pariquera-Açu, } \\
\text { SP, Brazil }\end{array}$ & $\begin{array}{l}24^{\circ} 36^{\prime} 41^{\prime \prime} \mathrm{S} \\
47^{\circ} 53^{\prime} 23^{\prime \prime} \mathrm{W}\end{array}$ & I, X- 2000 & D. Navia \\
\hline & & $\mathrm{L}$ & $\begin{array}{l}\text { Teresópolis, } \\
\text { RJ, Brazil }\end{array}$ & $\begin{array}{l}22^{\circ} 29^{\prime} 23^{\prime \prime} \mathrm{S} ; \\
42^{\circ} 54^{\prime} 47^{\prime \prime} \mathrm{W}\end{array}$ & III-2001 & D. Navia \\
\hline & & $\begin{array}{l}\mathrm{L}, \\
\mathrm{TS} \\
2\end{array}$ & $\begin{array}{l}\text { Cananéia, SP, } \\
\text { Brazil }\end{array}$ & $\begin{array}{l}24^{\circ} 53^{\prime} 45^{\prime \prime} \mathrm{S} \\
47^{\circ} 50^{\prime} 17^{\prime \prime} \mathrm{W}\end{array}$ & $\mathrm{I}, \mathrm{X}-2000$ & D. Navia \\
\hline & Bactris vulgaris ${ }^{1}$ & $\mathrm{~L}$ & $\begin{array}{l}\text { Pariquera-Açu, } \\
\text { SP, Brazil }\end{array}$ & $\begin{array}{l}24^{\circ} 36^{\prime} 41^{\prime \prime} \mathrm{S} \\
47^{\circ} 53^{\prime} 23^{\prime \prime} \mathrm{W}\end{array}$ & $\mathrm{I}-2001$ & D. Navia \\
\hline \multirow[t]{2}{*}{ Retracrus elaeis } & Chamaedorea costaricana ${ }^{1}$ & $\mathrm{~L}$ & $\begin{array}{l}\text { San José, Costa } \\
\text { Rica }\end{array}$ & $\begin{array}{l}09^{\circ} 56^{\prime} 16^{\prime \prime} \mathrm{N} \\
84^{\circ} 06^{\prime} 55^{\prime \prime} \mathrm{W}\end{array}$ & II-2003 & H. Aguilar \\
\hline & Elaeis guineensis & $\mathrm{L}$ & $\begin{array}{l}\text { Puntarenas, } \\
\text { Costa Rica }\end{array}$ & $\begin{array}{l}09^{\circ} 57^{\prime} 19^{\prime \prime} \mathrm{N} \\
85^{\circ} 02^{\prime} 00^{\prime \prime} \mathrm{W}\end{array}$ & V-2003 & H. Aguilar \\
\hline \multirow[t]{6}{*}{ Retracrus johnstoni } & Astrocaryum aculeatissimum ${ }^{1}$ & $\mathrm{~L}$ & $\begin{array}{l}\text { Pariquera-Açu, } \\
\text { SP, Brazil }\end{array}$ & $\begin{array}{l}24^{\circ} 36^{\prime} 41^{\prime \prime} \mathrm{S} \\
47^{\circ} 53^{\prime} 23^{\prime \prime} \mathrm{W}\end{array}$ & IV-1998 & M. G. C. Gondim Jr. \\
\hline & Bactris gasipaes ${ }^{1}$ & $\mathrm{~L}$ & $\begin{array}{l}\text { Pariquera-Açu, } \\
\text { SP, Brazil }\end{array}$ & $\begin{array}{l}24^{\circ} 36^{\prime} 41^{\prime \prime} \mathrm{S} \\
47^{\circ} 53^{\prime} 23^{\prime \prime} \mathrm{W}\end{array}$ & IV-1998 & M. G. C. Gondim Jr. \\
\hline & & $\mathrm{L}$ & $\begin{array}{l}\text { Limon, Costa } \\
\text { Rica }^{3}\end{array}$ & & IX-2003 & H. Aguilar \\
\hline & Bactris setosa $a^{1}$ & $\mathrm{~L}$ & $\begin{array}{l}\text { Cananéia, SP, } \\
\text { Brazil }\end{array}$ & $\begin{array}{l}24^{\circ} 53^{\prime} 45^{\prime \prime} \mathrm{S} \\
47^{\circ} 50^{\prime} 17^{\prime \prime} \mathrm{W}\end{array}$ & X-1998 & M. G. C. Gondim Jr. \\
\hline & & $\mathrm{L}$ & $\begin{array}{l}\text { Pariquera-Açu, } \\
\text { SP, Brazil }\end{array}$ & $\begin{array}{l}24^{\circ} 36^{\prime} 41^{\prime \prime} \mathrm{S} \\
47^{\circ} 53^{\prime} 23^{\prime \prime} \mathrm{W}\end{array}$ & IV-1998 & M. G. C. Gondim Jr. \\
\hline & & $\mathrm{L}$ & $\begin{array}{l}\text { Pariquera-Açu, } \\
\text { SP, Brazil }\end{array}$ & $\begin{array}{l}24^{\circ} 36^{\prime} 41^{\prime \prime} \mathrm{S} ; \\
47^{\circ} 53^{\prime} 23^{\prime \prime} \mathrm{W}\end{array}$ & $X-2000$ & D. Navia \\
\hline
\end{tabular}




\begin{tabular}{|c|c|c|c|c|c|}
\hline \multirow[t]{2}{*}{ Cocos nucifera } & $\mathrm{L}$ & $\begin{array}{l}\text { Cananéia, SP, } \\
\text { Brazil }\end{array}$ & $\begin{array}{l}24^{\circ} 53^{\prime} 45^{\prime \prime} \mathrm{S} ; \\
47^{\circ} 50^{\prime} 17^{\prime \prime} \mathrm{W}\end{array}$ & X-1998 & M. G. C. Gondim Jr. \\
\hline & $\mathrm{L}$ & $\begin{array}{l}\text { Piracicaba, SP, } \\
\text { Brazil }\end{array}$ & $\begin{array}{l}22^{\circ} 42^{\prime} 30^{\prime \prime} \mathrm{S} ; \\
47^{\circ} 48^{\prime} 00^{\prime \prime} \mathrm{W}\end{array}$ & IV, X-1998 & M. G. C. Gondim Jr. \\
\hline \multirow[t]{4}{*}{ Euterpe edulis ${ }^{1}$} & $\mathrm{~L}$ & $\begin{array}{l}\text { Cananéia, SP, } \\
\text { Brazil }\end{array}$ & $\begin{array}{l}24^{\circ} 53^{\prime} 45^{\prime \prime} \mathrm{S} ; \\
47^{\circ} 50^{\prime} 17^{\prime \prime} \mathrm{W}\end{array}$ & IV, X-1998 & M. G. C. Gondim Jr. \\
\hline & $\mathrm{L}$ & $\begin{array}{l}\text { Pariquera-Açu, } \\
\text { SP, Brazil }\end{array}$ & $\begin{array}{l}24^{\circ} 36^{\prime} 41^{\prime \prime} \mathrm{S} ; \\
47^{\circ} 53^{\prime} 23^{\prime \prime} \mathrm{W}\end{array}$ & IV-1998 & M. G. C. Gondim Jr. \\
\hline & $\mathrm{L}$ & $\begin{array}{l}\text { Piracicaba, SP, } \\
\text { Brazil }\end{array}$ & $\begin{array}{l}22^{\circ} 42^{\prime} 30^{\prime \prime} \mathrm{S}, \\
47^{\circ} 38^{\prime} 00^{\prime \prime} \mathrm{W}\end{array}$ & XI-2000 & D. Navia \\
\hline & $\mathrm{L}$ & $\begin{array}{l}\text { Jundiaí, SP, } \\
\text { Brazil }\end{array}$ & $\begin{array}{l}23^{\circ} 13^{\prime} 18^{\prime \prime} \mathrm{S} ; \\
46^{\circ} 55^{\prime} 16^{\prime \prime} \mathrm{W}\end{array}$ & $\mathrm{I}-2001$ & D. Navia \\
\hline \multirow[t]{2}{*}{ Elaeis guineensis ${ }^{1}$} & $\mathrm{~L}$ & $\begin{array}{l}\text { Igarassu, PE, } \\
\text { Brazil }\end{array}$ & $\begin{array}{l}27^{\circ} 53^{\prime} 28^{\prime \prime} \mathrm{S} \\
34^{\circ} 58^{\prime} 35^{\prime \prime} \mathrm{W}\end{array}$ & $\begin{array}{l}\text { VI-1998; I- } \\
1999\end{array}$ & M. G. C. Gondim Jr. \\
\hline & $\mathrm{L}$ & $\begin{array}{l}\text { Recife, PE, } \\
\text { Brazil }\end{array}$ & $\begin{array}{l}08^{\circ} 10^{\prime} 52^{\prime \prime} \mathrm{S} ; \\
34^{\circ} 54^{\prime} 47^{\prime \prime} \mathrm{W}\end{array}$ & $\begin{array}{l}\text { VI-1998; I- } \\
1999\end{array}$ & M. G. C. Gondim Jr. \\
\hline Euterpe oleracea $^{1}$ & $\mathrm{~L}$ & $\begin{array}{l}\text { Piracicaba, SP, } \\
\text { Brazil }\end{array}$ & $\begin{array}{l}22^{\circ} 42^{\prime} 30^{\prime \prime} \mathrm{S}, \\
47^{\circ} 38^{\prime} 00^{\prime \prime} \mathrm{W}\end{array}$ & $X-2000$ & D. Navia \\
\hline Euterpe precatoria ${ }^{1}$ & $\mathrm{~L}$ & $\begin{array}{l}\text { Manaus, AM, } \\
\text { Brazil }\end{array}$ & $\begin{array}{l}03^{\circ} 08^{\prime} 07^{\prime \prime} \mathrm{S} \\
60^{\circ} 01^{\prime} 34^{\prime \prime} \mathrm{W}\end{array}$ & X-2003 & D. Navia \\
\hline \multirow[t]{2}{*}{ Euterpe sp. } & $\mathrm{L}$ & $\begin{array}{l}\text { Igarassu, PE, } \\
\text { Brazil }\end{array}$ & $\begin{array}{l}27^{\circ} 53^{\prime} 28^{\prime \prime} \mathrm{S} \\
34^{\circ} 58^{\prime} 35^{\prime \prime} \mathrm{W}\end{array}$ & $\begin{array}{l}\text { VI-1998; I- } \\
1999\end{array}$ & M. G. C. Gondim Jr. \\
\hline & $\mathrm{L}$ & $\begin{array}{l}\text { Recife, PE, } \\
\text { Brazil }\end{array}$ & $\begin{array}{l}08^{\circ} 10^{\prime} 52^{\prime \prime} \mathrm{S} \\
34^{\circ} 54^{\prime} 47^{\prime \prime} \mathrm{W}\end{array}$ & $\begin{array}{l}\text { VI-1998; I- } \\
1999\end{array}$ & M. G. C. Gondim Jr. \\
\hline \multirow[t]{2}{*}{ Geonoma gamiovora ${ }^{1}$} & $\mathrm{~L}$ & $\begin{array}{l}\text { Pariquera-Açu, } \\
\text { SP, Brazil }\end{array}$ & $\begin{array}{l}24^{\circ} 36^{\prime} 41^{\prime \prime} \mathrm{S} ; \\
47^{\circ} 53^{\prime} 23^{\prime \prime} \mathrm{W}\end{array}$ & IV, X-1998 & M. G. C. Gondim Jr \\
\hline & $\mathrm{L}$ & $\begin{array}{l}\text { Pariquera-Açu, } \\
\text { SP, Brazil }\end{array}$ & $\begin{array}{l}24^{\circ} 36^{\prime} 41^{\prime \prime} \mathrm{S} ; \\
47^{\circ} 53^{\prime} 23^{\prime \prime} \mathrm{W}\end{array}$ & VIII-2000 & D. Navia \\
\hline Geonoma pohliana ${ }^{1}$ & $\mathrm{~L}$ & $\begin{array}{l}\text { Pariquera-Açu, } \\
\text { SP, Brazil }\end{array}$ & $\begin{array}{l}24^{\circ} 36^{\prime} 41^{\prime \prime} \mathrm{S} ; \\
47^{\circ} 53^{\prime} 23^{\prime \prime} \mathrm{W}\end{array}$ & IV, X-1998 & M. G. C. Gondim Jr. \\
\hline \multirow[t]{3}{*}{ Geonoma schottiana $^{1}$} & $\mathrm{~L}$ & $\begin{array}{l}\text { Cananéia, SP, } \\
\text { Brazil }\end{array}$ & $\begin{array}{l}24^{\circ} 53 ' 45^{\prime \prime} \mathrm{S} \\
47^{\circ} 50^{\prime} 17^{\prime \prime} \mathrm{W}\end{array}$ & X-1998 & M. G. C. Gondim Jr. \\
\hline & $\mathrm{L}$ & $\begin{array}{l}\text { Cananéia, SP, } \\
\text { Brazil }\end{array}$ & $\begin{array}{l}24^{\circ} 53^{\prime} 45^{\prime \prime} \mathrm{S} \\
47^{\circ} 50^{\prime} 17^{\prime \prime} \mathrm{W}\end{array}$ & V, X- 2000 & D. Navia \\
\hline & $\mathrm{L}$ & $\begin{array}{l}\text { Pariquera-Açu, } \\
\text { SP, Brazil }\end{array}$ & $\begin{array}{l}24^{\circ} 36^{\prime} 41^{\prime \prime} \mathrm{S} \\
47^{\circ} 53^{\prime} 23^{\prime \prime} \mathrm{W}\end{array}$ & IV-2000 & D. Navia \\
\hline Mauritia flexuosa $^{1}$ & $\mathrm{~L}$ & $\begin{array}{l}\text { Manaus, AM, } \\
\text { Brazil }\end{array}$ & $\begin{array}{l}03^{\circ} 08^{\prime} 07^{\prime \prime} \mathrm{S} \\
60^{\circ} 01^{\prime} 34^{\prime \prime} \mathrm{W}\end{array}$ & X-2003 & D. Navia \\
\hline Scheelea sp. & $\mathrm{L}$ & $\begin{array}{l}\text { Campinas, SP, } \\
\text { Brazil }\end{array}$ & $\begin{array}{l}24^{\circ} 36^{\prime} 41^{\prime \prime} \mathrm{S}, \\
47^{\circ} 53^{\prime} 23^{\prime \prime} \mathrm{W}\end{array}$ & V-2000 & D. Navia \\
\hline \multirow[t]{2}{*}{ Syagrus. romanzoffiana ${ }^{1}$} & $\mathrm{~L}$ & $\begin{array}{l}\text { Pariquera-Açu, } \\
\text { SP, Brazil }\end{array}$ & $\begin{array}{l}24^{\circ} 36^{\prime} 41^{\prime \prime} \mathrm{S} ; \\
47^{\circ} 53^{\prime} 23^{\prime \prime} \mathrm{W}\end{array}$ & IV, X-1998 & M. G. C. Gondim Jr. \\
\hline & $\mathrm{L}$ & $\begin{array}{l}\text { Piracicaba, SP, } \\
\text { Brazil }\end{array}$ & $\begin{array}{l}22^{\circ} 42^{\prime} 30^{\prime \prime} \mathrm{S} \\
47^{\circ} 48^{\prime} 00^{\prime \prime} \mathrm{W}\end{array}$ & IV, X-1998 & M. G. C. Gondim Jr. \\
\hline
\end{tabular}

Table 2 summarizes the eriophyoids found on different parts of each palm tree species, with the corresponding first report per country, with additional new reports from the present survey.

Details about type locality and type host, further records and damage of each eriophyoid species listed in Table 2 are provided hereafter. Until the moment, all Eriophyoidea mites associated with palm plants are vagrant. 
TABLE 2. Eriophyoid mites associated with palm trees around the world (infested plant parts: L — leaves; TS — terminal shoot; F - fruits).

\begin{tabular}{|c|c|c|c|c|}
\hline Palm Host & Eriophyoidea Mite & $\begin{array}{l}\text { Plant } \\
\text { Part }\end{array}$ & Country & Reference \\
\hline \multirow[t]{3}{*}{ Acrocomia aculeata } & Aceria biornata & TS & Brazil & Navia \& Flechtmann 2002 \\
\hline & Notostrix acuminata & $\mathrm{L}$ & Brazil & Navia \& Flechtmann 2003a \\
\hline & Notostrix attenuata & $\mathrm{L}$ & Brazil & This publication \\
\hline \multirow[t]{2}{*}{ Aiphanes sp. } & Propilus gentyi & $\mathrm{L}$ & Colombia & Keifer $1975 b$ \\
\hline & Propilus spinosus & $\mathrm{L}$ & Colombia & Keifer $1975 b$ \\
\hline Arenga englera & Epitrimerus englerus & $\mathrm{L}$ & Taiwan & Huang 2001 \\
\hline Astrocaryum acaule & Palmiphytoptus barbosae & $\mathrm{L}$ & Brazil & Navia \& Flechtmann 2005 \\
\hline \multirow[t]{4}{*}{ Astrocaryum aculeatissimum } & Retracrus johnstoni & $\mathrm{L}$ & Brazil & This publication \\
\hline & Palmiphytoptus oculatus & $\mathrm{L}$ & Brazil & Navia \& Flechtmann 2002 \\
\hline & Propilus gentyi & $\mathrm{L}$ & Brazil & This publication \\
\hline & Propilus syagris & $\mathrm{L}, \mathrm{TS}$ & Brazil & This publication \\
\hline \multirow[t]{3}{*}{ Attalea dubia } & Aceria translinea & $\mathrm{TS}$ & Brazil & Navia \& Flechtmann 2002 \\
\hline & Notostrix fissipes & $\mathrm{L}$ & Brazil & Navia \& Flechtmann 2003a \\
\hline & Notostrix nasutiformes & $\mathrm{L}$ & Brazil & Gondim Jr. et al. 2000 \\
\hline \multirow[t]{2}{*}{ Attalea geraensis } & Amrineus cocofolius & $\mathrm{L}$ & Brazil & This publication \\
\hline & Notostrix butiae & $\mathrm{L}$ & Brazil & This publication \\
\hline Attalea phalerata & Notostrix nasutiformes & $\mathrm{L}$ & Brazil & Gondim Jr. et al. 2000 \\
\hline Attalea sp. & Notostrix jamaicae & $\mathrm{L}$ & Brazil & Santana \& Flechtmann 1998 \\
\hline Bactris ferruginea & Notostrix nasutiformes & $\mathrm{L}$ & Brazil & Gondim Jr. et al. 2000 \\
\hline \multirow[t]{4}{*}{ Bactris gasipaes } & Notostrix nasutiformes & $\mathrm{L}$ & Brazil & Gondim Jr. et al. 2000 \\
\hline & Retracrus elaeis & $\mathrm{L}$ & Costa Rica & Ochoa et al. 1994 \\
\hline & Retracrus johnstoni & $\mathrm{L}$ & Brazil & This publication \\
\hline & Retracrus johnstoni & $\mathrm{L}$ & Costa Rica & This publication \\
\hline \multirow[t]{6}{*}{ Bactris setosa } & Notostrix exigua & $\mathrm{L}, \mathrm{TS}$ & Brazil & This publication \\
\hline & Notostrix miniseta & $\mathrm{L}$ & Brazil & Navia \& Flechtmann 2003a \\
\hline & Notostrix nasutiformes & $\mathrm{L}$ & Brazil & Gondim Jr. et al. 2000 \\
\hline & Palmiphytoptus oculatus & $\mathrm{TS}$ & Brazil & Navia \& Flechtmann 2002 \\
\hline & Propilus spinosus & $\mathrm{L}$ & Brazil & This publication \\
\hline & Retracrus johnstoni & $\mathrm{L}$ & Brazil & This publication \\
\hline \multirow[t]{3}{*}{ Bactris vulgaris } & Notostrix miniseta & $\mathrm{L}$ & Brazil & Navia \& Flechtmann 2003a \\
\hline & Propilus gentyi & $\mathrm{L}$ & Brazil & This publication \\
\hline & Propilus syagris & $\mathrm{L}$ & Brazil & This publication \\
\hline \multirow[t]{6}{*}{ Borassus flabellifer } & Aceria guerreronis & $\mathrm{F}$ & India & Ramaraju \& Rabindra 2002 \\
\hline & Aceria guerreronis & $\mathrm{F}$ & Sri Lanka & Moraes \& Fernando ${ }^{a}$ \\
\hline & Mackiella borasis & $\mathrm{TS}$ & India & Mohanasundaram 1981 \\
\hline & Neocupacarus flabelliferis & $\mathrm{L}$ & India & Das \& Chakrabarti 1985 \\
\hline & Neodialox palmyrae & $\mathrm{L}$ & India & Mohanasundaram 1983 \\
\hline & Notostrix flabelliferea & $\mathrm{L}$ & India & Mohanasundaram 1982 \\
\hline \multirow[t]{2}{*}{ Butia archeri } & Amrineus cocofolius & $\mathrm{L}$ & Brazil & This publication \\
\hline & Notostrix butiae & $\mathrm{L}, \mathrm{TS}$ & Brazil & This publication \\
\hline
\end{tabular}




\begin{tabular}{|c|c|c|c|c|}
\hline & Notostrix longiseta & $\mathrm{L}$ & Brazil & Navia \& Flechtmann 2003a \\
\hline Butia capitata & Notostrix butiae & $\mathrm{L}$ & Brazil & This publication \\
\hline \multirow[t]{3}{*}{ Butia eriospatha } & Amrineus cocofolius & $\mathrm{L}$ & Brazil & This publication \\
\hline & Notostrix butiae & $\mathrm{L}$ & Brazil & Gondim Jr. et al. 2000 \\
\hline & Proartacris longior & $\mathrm{TS}$ & Brazil & Navia \& Flechtmann 2002 \\
\hline Calamus australis & Epitrimerus calami & $\mathrm{L}$ & Australia & Keifer 1969 \\
\hline \multirow[t]{2}{*}{ Chamaedorea costaricana } & Retracrus elaeis & $\mathrm{L}$ & Costa Rica & This publication \\
\hline & Retracrus johnstoni & $\mathrm{L}$ & Costa Rica & Ochoa et al. 1994 \\
\hline Chamaedorea elegans & Retracrus johnstoni & $\mathrm{L}$ & Costa Rica & Schliesske 1988 \\
\hline \multirow[t]{2}{*}{ Chamaedorea sp. } & Retracrus johnstoni & $\mathrm{L}$ & Mexico & Keifer 1965 \\
\hline & Retracrus johnstoni & $\mathrm{L}$ & Costa Rica & Ochoa et al. 1994 \\
\hline \multirow[t]{4}{*}{ Chamaerops humilis } & Adenoptus chamaeropsi & $?$ & Ukraine & Mitrofanov et al. 1983 \\
\hline & Adenoptus migeoni & $\mathrm{L}$ & France & Navia \& Flechtmann 2003b \\
\hline & Epitrimerus steveni & $\mathrm{L}$ & Ukraine & Mitrofanov et al. 1983 \\
\hline & Gilarovella caniculata & $\mathrm{L}$ & Ukraine & Mitrofanov et al. 1983 \\
\hline \multirow[t]{21}{*}{ Cocos nucifera } & Acathrix trymatus & $\mathrm{L}(?), \mathrm{TS}$ & Phillipines, & Keifer $1962 b$ \\
\hline & Acathrix trymatus & $\mathrm{L}(?), \mathrm{TS}$ & USA & Amrine Jr. \& de Lillo 2003 \\
\hline & Aceria guerreronis & $\mathrm{F}$ & America & Keifer 1965 \\
\hline & Aceria guerreronis & $\mathrm{F}$ & $\begin{array}{l}\text { Africa } \\
\text { (São Tomé } \\
\text { and Principe) }\end{array}$ & Cabral \& Carmona 1968 \\
\hline & Aceria guerreronis & $\mathrm{F}$ & $\begin{array}{l}\text { Asia } \\
\text { (India, } \\
\text { SriLanka) }\end{array}$ & $\begin{array}{l}\text { Sathiamma et al. 1998; Fernando } \\
\text { et al. } 2002\end{array}$ \\
\hline & Aceria guerreronis & $\mathrm{TS}$ & Brazil & Aquino \& Arruda 1967 \\
\hline & Acritonotus denmarki & $\mathrm{L}$ & USA & Moore \& Howard 1996 \\
\hline & Amrineus cocofolius & $\mathrm{L}$ & Brazil & $\begin{array}{l}\text { Flechtmann 1994, 1997; Santana } \\
\text { and Flechtmann 1998; Navia et } \\
\text { al. } 2005\end{array}$ \\
\hline & Amrineus cocofolius & $\mathrm{F}$ & Brazil & Ferreira et al. 2001 \\
\hline & Amrineus cocofolius & $?$ & Brazil & $\begin{array}{l}\text { Flechtmann 1997; Santana \& } \\
\text { Flechtmann 1998; Navia et al. } \\
2005\end{array}$ \\
\hline & Amrineus cocofolius & $\mathrm{F}$ & Mexico & Navia et al. 2005 \\
\hline & Amrineus coconuciferae & $\mathrm{L}$ & USA & Keifer 1962a \\
\hline & $\begin{array}{l}\text { Colomerus novahebriden- } \\
\text { sis }\end{array}$ & $\mathrm{F}$ & $\begin{array}{l}\text { Southeast } \\
\text { Asia, Oce- } \\
\text { ania }\end{array}$ & $\begin{array}{l}\text { Keifer 1977; Hall et al. 1980; } \\
\text { Kang 1981; Moore \& Howard } \\
1996\end{array}$ \\
\hline & Dialox stellatus & $\mathrm{L}, \mathrm{F}$ & Phillipines & Keifer $1962 c$ \\
\hline & Epitrimerus elaeis & $?$ & Côte d'Ivoire & Amrine \& de Lillo 2003 \\
\hline & Nacerimina gutierrezi & $\mathrm{L}$ & Samoa & Keifer 1979 \\
\hline & Notostrix attenuata & $\mathrm{L}$ & Phillipines & Keifer 1963 \\
\hline & Notostrix attenuata & $\mathrm{L}$ & India & Mohanasundaram 1984 \\
\hline & Notostrix attenuata & $\mathrm{L}$ & Brazil & Flechtmann \& Santana 1997 \\
\hline & Notostrix jamaicae & $\mathrm{L}$ & Jamaica & Keifer 1970 \\
\hline & Notostrix jamaicae & $\mathrm{L}$ & Costa Rica & Schliesske 1998 \\
\hline
\end{tabular}




\begin{tabular}{|c|c|c|c|c|}
\hline & Notostrix nasutiformes & $\mathrm{L}$ & Brazil & Gondim Jr. et al. 2000 \\
\hline & Notostrix nasutiformes & $\mathrm{L}$ & Mexico & This publication \\
\hline & Retracrus elaeis & $\mathrm{L}$ & Colombia & Genty \& Reyes 1977 \\
\hline & Retracrus elaeis & $\mathrm{L}$ & Costa Rica & Schliesske 1988 \\
\hline & Retracrus johnstoni & $\mathrm{L}$ & Brazil & Santana et al. 1994 \\
\hline & Scolocenus spiniferus & $\mathrm{L}$ & Phillipines & Keifer $1962 c$ \\
\hline Collinia sp. & Calacarus palmae & $?$ & India & Mohanasundaram 1994 \\
\hline \multirow[t]{6}{*}{ Elaeis guineensis } & Epitrimerus elaeis & $\mathrm{L}$ & Côte d'Ivoire & Boczek \& Natcheff 1989 \\
\hline & Phyllocoptes mariaui & $\mathrm{L}$ & Côte d'Ivoire & Boczek \& Natcheff 1989 \\
\hline & Retracrus elaeis & $\mathrm{L}$ & Colombia & Keifer 1975a \\
\hline & Retracrus elaeis & $\mathrm{L}$ & Costa Rica & This publication \\
\hline & Retracrus johnstoni & $\mathrm{L}$ & Brazil & This publication \\
\hline & Tegonotus gutierrezi & $\mathrm{L}$ & Côte d'Ivoire & Boczek \& Natcheff 1989 \\
\hline Elaeis oleifera & Propilus tavaresi & $\mathrm{L}$ & Brazil & Navia \& Flechtmann 2005 \\
\hline \multirow[t]{4}{*}{ Euterpe edulis } & Davisella palmea & $\mathrm{L}$ & Brazil & This publication \\
\hline & Glabrisceles euterpis & $\mathrm{TS}$ & Brazil & Navia \& Flechtmann 2002 \\
\hline & Notostrix exigua & $\mathrm{L}$ & Brazil & Flechtmann 1998 \\
\hline & Retracrus johnstoni & $\mathrm{L}$ & Brazil & This publication \\
\hline Euterpe oleracea & Retracrus johnstoni & $\mathrm{L}$ & Brazil & This publication \\
\hline \multirow[t]{2}{*}{ Euterpe precatoria } & Euterpia fissa & $\mathrm{L}$ & Brazil & Navia \& Flechtmann 2005 \\
\hline & Retracrus johnstoni & $\mathrm{L}$ & Brazil & This publication \\
\hline \multirow[t]{2}{*}{ Euterpe sp. } & Notostrix exigua & $\mathrm{L}$ & Brazil & This publication \\
\hline & Retracrus johnstoni & $\mathrm{L}$ & Brazil & This publication \\
\hline Geonoma brevispatha & Acathrix lobata & $\mathrm{TS}$ & Brazil & Navia \& Flechtmann 2002 \\
\hline \multirow[t]{2}{*}{ Geonoma gamiovora } & Nasuchus pindobates & $\mathrm{L}$ & Brazil & Navia \& Flechtmann 2002 \\
\hline & Retracrus johnstoni & $\mathrm{L}$ & Brazil & This publication \\
\hline Geonoma pohliana & Retracrus johnstoni & $\mathrm{L}$ & Brazil & This publication \\
\hline \multirow[t]{3}{*}{ Geonoma schottiana } & Acathrix lobata & $\mathrm{TS}, \mathrm{L}$ & Brazil & Navia \& Flechtmann 2002 \\
\hline & Knorella geonomae & $\mathrm{L}$ & Brazil & Gondim Jr. et al. 2000 \\
\hline & Retracrus johnstoni & $\mathrm{L}$ & Brazil & This publication \\
\hline Geonoma sp. & Acathrix lobata & $\mathrm{L}$ & Brazil & Navia \& Flechtmann 2002 \\
\hline Geonoma sp. & Schizacea geonomae & $\mathrm{L}$ & Brazil & Navia \& Flechtmann 2002 \\
\hline Livistona chinensis & Calepitrimerus livistonae & $?$ & China & Wei \& Feng 2002 \\
\hline \multirow[t]{2}{*}{ Lytocaryum hoehnei } & Notostrix trifida & $\mathrm{L}$ & Brazil & Navia \& Flechtmann 2003a \\
\hline & Propilus pellitus & $\mathrm{L}$ & Brazil & Navia \& Flechtmann 2002 \\
\hline Lytocaryum weddellianum & Aceria guerreronis & $\mathrm{TS}$ & Brazil & Flechtmann 1994 \\
\hline \multirow[t]{3}{*}{ Mauritia flexuosa } & Notostrix spinula & $\mathrm{L}$ & Brazil & Navia \& Flechtmann 2005 \\
\hline & Propilus alternatus & $\mathrm{L}$ & Brazil & Navia \& Flechtmann 2005 \\
\hline & Retracrus johnstoni & $\mathrm{L}$ & Brazil & This publication \\
\hline Phoenix canariensis & Tumescoptes phoenixi & $\mathrm{L}$ & South Africa & Meyer 1992 \\
\hline \multirow[t]{3}{*}{ Phoenix dactylifera } & Mackiella phoenicis & $\mathrm{TS}$ & USA & Keifer 1939a \\
\hline & Mackiella phoenicis & $?$ & Iraq & Mohamed \& El-Haidari 1968 \\
\hline & Tumescoptes trachycarpi & $?$ & Iraq & Mohamed \& El-Haidari 1968 \\
\hline Phoenix reclinata & Tumescoptes dicrus & $\mathrm{L}$ & South Africa & Meyer 1992 \\
\hline
\end{tabular}




\begin{tabular}{|c|c|c|c|c|}
\hline Roystonea elata & Acritonotus denmarki & $\mathrm{L}$ & USA & Keifer 1962c \\
\hline Roystonea sp. & Diptacus borinquensis & $\mathrm{L}$ & Puerto Rico & Cromroy 1958 \\
\hline Sabal sp. & Notostrix vazquezae & $\mathrm{L}$ & Mexico & Navia \& Flechtmann 2003a \\
\hline Scheelea sp. & Retracrus johnstoni & $\mathrm{L}$ & Brazil & This publication \\
\hline Syagrus cocoides & Aceria gymnoscuta & $\mathrm{F}$ & Brazil & Navia \& Flechtmann 2002 \\
\hline Syagrus flexuosa & Aceria gymnoscuta & $\mathrm{L}$ & Brazil & Navia \& Flechtmann 2002 \\
\hline Syagrus microphylla & Notostrix butiae & $\mathrm{L}$ & Brazil & This publication \\
\hline \multirow[t]{2}{*}{ Syagrus oleracea } & Notostrix acuminata & $\mathrm{L}$ & Brazil & Navia \& Flechtmann 2003a \\
\hline & Aceria gymnoscuta & $\mathrm{L}$ & Brazil & Bellini et al. 2005 \\
\hline Syagrus quinquefaria & Notostrix butiae & $\mathrm{L}$ & Brazil & This publication \\
\hline \multirow[t]{9}{*}{ Syagrus romanzoffiana } & Aceria guerreronis & $\mathrm{TS}$ & USA & Ansaloni \& Perring 2002 \\
\hline & Aceria translinea & $\mathrm{TS}$ & Brazil & Navia \& Flechtmann 2002 \\
\hline & Amrineus cocofolius & $\mathrm{F}$ & Brazil & This publication \\
\hline & Davisella palmea & $\mathrm{L}$ & Brazil & Flechtmann 1998 \\
\hline & Notostrix attenuata & $\mathrm{L}$ & Brazil & This publication \\
\hline & Notostrix jamaicae & $\mathrm{L}$ & Brazil & Santana \& Flechtmann 1998 \\
\hline & Propilus spinosus & $\mathrm{L}$ & Brazil & This publication \\
\hline & Propilus syagris & $\mathrm{L}$ & Brazil & Gondim Jr. et al. 2000 \\
\hline & Retracrus johnstoni & $\mathrm{L}$ & Brazil & Santana \& Flechtmann 1998 \\
\hline Trachycarpus fortunei & Tumescoptes trachycarpi & $\mathrm{L}$ & China & Keifer 1939b; Kuang 1991 \\
\hline
\end{tabular}

a. Moraes, G.J.de. (Escola Superior de Agricultura Luiz de Queiroz (ESALQ), Universidade de São Paulo) \& Fernando, L.C.P (Coconut Research Institute, Lunuwila, Sri Lanka) (in preparation).

\section{Diptilomiopidae}

\section{Diptilomiopinae}

\section{Davisella palmea (Flechtmann, 1998)}

Rhynacus palmeus Flechtmann, 1998

Davisella palmea $\mathrm{n}$. comb. (Amrine, Stasny \& Flechtmann 2003)

Type host: Syagrus romanzoffiana (Cham.) Glassm.

Type locality: Brazil (Itatiaia, Rio de Janeiro)

Other host: Euterpe edulis Mart. (this publication)

Plant part: upper leaf surface

Remarks: collected in association with numerous specimens of a species of Retracrus Keifer, 1965 (Phytoptidae), both together on discolored areas of infested leaves (Flechtmann 1998).

\section{Dialox stellatus Keifer, 1962c}

Type host: Cocos nucifera $\mathrm{L}$.

Type locality: Philippines (Guinobatan, Albay)

Plant part: fruit, leaf (Briones \& Sill Jr. 1963)

Remarks: type species of this monospecific genus (Amrine Jr. \& de Lillo 2003). Produce star-like masses of whitish wax over the body (Keifer 1962c; Briones \& Sill Jr. 1963). Its possible action as vector of the virus that causes the coconut disease known as "cadang-cadang" in Philippines was investigated, with negative 
results. Found in small populations, apparently causing no damage (Briones \& Sill Jr. 1963; Moore \& Howard 1996).

\section{Diptacus borinquensis Cromroy, 1958}

Type host: Roystonea sp.

Type locality: Puerto Rico (Corozal)

Plant part: leaf

\section{Neodialox palmyrae Mohanasundaram, 1983}

Type host: Borassus flabellifer $\mathrm{L}$.

Type locality: India (Vriddhachalam, Tamil Nadu)

Plant part: leaf

Remarks: type species of this monospecific genus (Amrine Jr. \& de Lillo 2003).

\section{Eriophyidae}

\section{Cecidophyinae, Colomerini}

\section{Colomerus novahebridensis Keifer, 1977}

Type host: Cocos nucifera $\mathrm{L}$.

Type locality: Saraoutou, Santo and New Hebrides Islands

Other locality: India (Andaman and Nicobar Islands; Bangalore, Karnataka); widely distributed in Southeast Asia and Oceania (Hall et al. 1980; Kang 1981; Mallik et al. 2003)

Plant part: under bract of ripe fruit

Remarks: reported to cause damage to coconut in the Philippines (Moore \& Howard 1996), but not to affect yield in Malaysia (Kang 1981).

\section{Eriophyinae, Eriophyini}

\section{Aceria biornata Navia \& Flechtmann, 2002}

Type host: Acrocomia aculeata (Jacq.) Lodd.

Type locality: Brazil (Piracicaba, São Paulo)

Plant part: terminal shoot

\section{Aceria guerreronis Keifer, 1965}

Type host: Cocos nucifera L.

Type locality: Mexico (Guerrero)

Other host: Borassus flabellifer L. (Ramaraju \& Rabindra 2002); Lytocaryum weddellianum (H. Wendl.)

Tol. (Flechtmann 1989); Syagrus romanzoffiana (Cham.) Glass. (Ansaloni \& Perring 2002)

Other locality: widely distributed in America and Africa; Asia (India and Sri Lanka)

Plant part: under the bract of fruit; leaf; terminal shoot

Remarks: Aceria guerreronis, the coconut mite, is an invasive species that became a key pest of coconut (Cocos nucifera L.) in the Americas, Africa and recently in Indo-Ocean countries. Infestations by A. guerreronis in coconut fruits cause physical damage to growing tissues, which become necrotic and suberized, resulting in uneven growth and premature drop (Doreste 1968; Moore \& Howard 1996). In Mexico, in the same 
year of its description, an infestation varying from 80 to $100 \%$ had been observed in several regions of the country, indicating that the mite had already been disseminated in that country (Ortega et al. 1965). Collection records indicate that A. guerreronis was also already present in other regions from South-America even before its original description. Symptoms similar to that caused by coconut mite infestations were observed before 1965 in Colombia, Venezuela and Brazil (Robbs \& Peracchi 1965; Doreste 1968; Zuluaga \& Sánchez 1971). In Colombia, it was reported in 1948 the occurrence of a symptom named as "mancha de la nuez del cocotero" in several areas of the Atlantic coast (Zuluaga \& Sánchez 1971). This symptom had also been observed by Sánchez (1962) in Zuluaga \& Sánchez (1971), who denominated it as "roña o escoriación de los frutos del cocotero". The causal agent of this symptom had not been determined until affected fruits were examined in detail and mites were found, identified as A. guerreronis.

In Brazil, Robbs \& Peracchi (1965) reported A. guerreronis for the first time, infesting coconut in Santa Cruz, State of Rio de Janeiro, causing "russeting" and premature fruit drop. These authors mention that the same symptons had been observed before in State of Pernambuco. In Brazil, in addition to infesting fruits, $A$. guerreronis was also reported to cause the death of buds of young coconut plants (Aquino \& Arruda 1967, Aquino et al. 1968). However, this type of damage has not been seen since A. guerreronis was also reported infesting terminal shoots of Lytocaryum weddellianum (H. Wendl.) Tol. in the State of São Paulo, Brazil (Flechtmann 1989).

In 1967, a significantly premature drop of coconut fruits was observed in the State of Zulia, Venezuela, caused by high infestations of A. guerreronis. This was the first report of the coconut mite in that country. Afterwards it was observed that the mite was already widely disseminated in all regions of the Maracaibo Lake, Northwest of Venezuela. According to the farmers the problem emerged in 1965/1966 with losses reaching $70 \%$ of fruits in that region (Doreste 1968).

In the Caribbean region the presence of the coconut mite was first reported in Cuba during the early 1970's, in the Baracoa region where the coconut production area is concentrated (Estrada \& Gonzalez 1975). The intensity of infestation was from 42 to $65 \%$ (Suarez 1991). In Trinidad, the exact year of occurrence of the coconut mite is unknown, however it was around 1976 (Griffith 1984). In Puerto Rico, coconut mite infestations were observed since 1977 (Howard et al. 1990); in Saint Lucie since 1980 (Moore et al. 1989); in Grenada and Saint Vincent before 1985 and in Republica Dominicana since 1984 (Moore 1986). In Costa Rica the first report of A. guerreronis occurrence is from Schliesske (1988), who considered the species as endemic, because in the Atlantic coast, South of Province of Limon, $90 \%$ of coconut palms were infested and a high percentage of fruits were damaged.

In the USA, the presence of A. guerreronis was reported for the first time in Florida, in 1984 (Howard et al. 1990). In 1997 infestations of A. guerreronis in young Queen palm plants, S. romanzoffiana (Cham.) Glass. were reported in San Diego, California, causing the death of terminal shoots (Ansaloni \& Perring 2002).

Almost simultaneously with its original description from Mexico and the reports in South American countries - Brazil, Colombia and Venezuela - the coconut mite was reported from Africa. It was found on the São Tomé and Principe Islands off the West coast of Africa in the Gulf of Guinea in 1966 (Cabral \& Carmona 1968). In continental Africa, A. guerreronis was reported for the first time in Benin, in 1967, and in less than two years the mite was found in all coconut producing areas in this country (Mariau 1969, 1977). Then the coconut mite was found in neighbouring countries - Togo, Nigeria and Cameroon (Mariau 1977; Julia \& Mariau 1979). In Côte d'Ivoire (Ivory Coast) it was first reported in 1975, in several Southeastern localities, causing a reduction of 7 to $15 \%$ in copra production (Julia \& Mariau 1979). Later damage by A. guerreronis was observed in Gambia, indicating that the mite is also present in all countries between Gambia and Togo (Moore \& Howard 1996).

In East Africa A. guerreronis was found in Tanzania in the early 1980's and today is widely disseminated in the coconut production areas of this country as well as off the coast - Mafia, Zanzibar and Pemba Islands. During surveys conducted in affected areas from 1992 to 1996, the losses in coconut dry weight reached 20 to 
$30 \%$ and due to premature dropping were on average $21 \%$ (Seguni 2000).

The most recent records of the coconut mite were in Asia, more specifically in India and Sri Lanka, where the species was unknown until the end of the 1990s (Sathiamma et al. 1998; Fernando et al. 2002). In 1997 A. guerreronis infestations were observed in Sri Lanka. The mite pest was found in Kalpitiya, disseminating to neighbouring areas of about 15.000 acres. The incidence varied from 5 to $100 \%$ (Fernando et al. 2002). In India, a high infestation was reported in 1998, in the District of Ernakulam, Central Kerala (Sathiamma et al. 1998) and in 1999 surveys showed that the pest was already stablished in all State of Kerala and also in some areas of Tamil Nadu, Karnataka and Andra Pradesh, in South India. Aceria guerreronis occurrence was also observed in the west islands of Lakshadweep - Minicoy, Kalpeni and Kavaratti (Haq 1999) and in the east islands - Adamans and Nicobar (Prasad \& Raganath 2000). In West India, Orissa, the coconut mite was reported for the first time in 2000 (Jagadiswari et al. 2001) and in East India, in Gujarat, in 2003 (Desai et al. 2003). Aceria guerreronis has also been found infesting Palmyra palm, Borassus flabellifer L., in Coimbatore, Tamil Nadu and Sirugamani, India and Sri Lanka (Ramaraju \& Rabindra 2002; Subramanian 2002; Moraes \& Fernando [Moraes, G.J.de. (Escola Superior de Agricultura Luiz de Queiroz (ESALQ), Universidade de São Paulo) \& Fernando, L.C.P (Coconut Research Institute, Lunuwila, Sri Lanka) (in preparation)].

\section{Aceria gymnoscuta Navia \& Flechtmann, 2002}

Type host: Syagrus flexuosa (Mart.) Becc.

Type locality: Brazil (Jundiaí, São Paulo)

Other host: Syagrus cocoides Mart. (Navia \& Flechtmann 2002); Syagrus oleracea (Mart.) Becc. (Bellini et al. 2005)

Plant part: upper leaf surface; under bract of fruit

Remarks: slight variations in morphological characters were observed in specimens collected from Syagrus oleracea (Mart.) Becc. (Bellini et al. 2005).

\section{Aceria translinea Navia \& Flechtmann, 2002}

Type host: Attalea dubia (Mart.) Burret.

Type locality: Brazil (Pariquera-Açu, São Paulo)

Other host: Syagrus romanzoffiana (Cham.) Glassm. (Navia \& Flechtmann 2002)

Plant part: terminal shoot

\section{Nacerimina gutierrezi Keifer, 1979}

Type host: Cocos nucifera $\mathrm{L}$.

Type locality: Samoa (Pago Pago, Tuituila)

Plant part: leaf

Remarks: type species of the genus (Amrine Jr. \& de Lillo 2003). Found among trichomes of leaflet midrib (Keifer 1979).

\section{Proartacris longior Navia \& Flechtmann, 2002}

Type host: Butia eriospatha (Mart.) Becc.

Type locality: Brazil (Piracicaba, São Paulo)

Plant part: terminal shoot

\section{Phyllocoptinae, Acaricalini}

Knorella geonomae Gondim Jr., Flechtmann \& Moraes, 2000 
Type host: Geonoma schottiana Mart.

Type locality: Brazil (Cananéia, São Paulo)

Plant part: upper leaf surface (Gondim Jr., Flechtmann \& Moraes 2000); terminal shoot (this publication)

\section{Nasuchus pindobates Navia \& Flechtmann, 2002}

Type host: Geonoma gamiovora Barb. Rodr.

Type locality: Brazil (Luiz Antônio, São Paulo)

Plant part: upper leaf surface

Remarks: type species of this monospecific genus (Amrine Jr. \& de Lillo 2003).

\section{Schizacea geonomae Navia \& Flechtmann, 2002}

Type host: Geonoma sp.

Type locality: Brazil (Rio de Janeiro, São Paulo)

Plant part: upper leaf surface

\section{Tumescoptes dicrus Meyer, 1992}

Type host: Phoenix reclinata Jacquin

Type locality: South Africa (Donkerhoek, Rustenberg)

Plant part: leaf

\section{Tumescoptes phoenixi Meyer, 1992}

Type host: Phoenix canariensis Hort. ex Chabaud

Type locality: South Africa (Pretoria, Gauteng)

Plant part: fold of young leaf

\section{Tumescoptes trachycarpi Keifer, 1939}

Type host: Trachycarpus fortunei (W. J. Hooker) H. Wendl.

Type locality: intercepted in San Francisco, California, USA

Other host: Phoenix dactylifera L. (Mohamed \& El-Haidari 1968)

Other locality: China (Kuang 1991); Iraq (Baghdad) (Mohamed \& El-Haidari 1968)

Plant part: leaf

Remarks: type species of the genus (Amrine Jr. \& de Lillo 2003).

\section{Phyllocoptinae, Anthocoptini}

\section{Notostrix acuminata Navia \& Flechtmann, 2003a}

Type host: Acrocomia aculeata (Jacquin) Lodd.

Type locality: Brazil (Piracicaba, São Paulo)

Other host: Syagrus oleracea (Mart.) Becc.

Plant part: upper leaf surface; terminal shoot

Remarks: body covered with white wax (Navia \& Flechtmann 2003a).

\section{Notostrix attenuata Keifer, 1963}

Type host: Cocos nucifera L.

Type locality: Philippines (Guinobatan, Albay) 
Other host: Acrocomia aculeata (Jacq.) Lodd.; Syagrus romanzoffiana (Cham.) Glassm. (this publication) Other locality: Brazil (Sergipe) (Flechtmann \& Santana 1997); India (Kerala) (Mohanasundaram 1984)

Plant part: leaf

Remarks: type species of the genus (Amrine Jr. \& de Lillo 2003).

\section{Notostrix butiae Gondim Jr., Flechtmann \& Moraes, 2000}

Type host: Butia eriospatha (Mart.) Becc.

Type locality: Brazil (Piracicaba, São Paulo)

Other host: Attalea geraensis Barb. Rodr.; Butia archeri (Glass.) Glass.; Butia capitata (Mart.) Becc.; Syagrus microphylla Burret; Syagrus quinquefaria Becc. (this publication)

Plant part: upper leaf surface (Gondim Jr., Flechtmann \& Moraes, 2000); terminal shoot (this publication)

Remarks: specimens from B. archeri, B. capitata and S. microphylla presented slightly longer setae, as well as shorter and wider epigynium than holotype (this publication).

\section{Notostrix exigua Flechtmann, 1998}

Type host: Euterpe edulis Mart.

Type locality: Brazil (Itatiaia, Rio de Janeiro)

Other host: Bactris setosa Mart.; Euterpe sp. (this publication)

Other locality: Brazil (Pernambuco; São Paulo) (this publication)

Plant part: upper leaf surface (Flechtmann, 1998); terminal shoot (this publication)

Remarks: seen as white wax stripes on leaf surface (this publication).

\section{Notostrix fissipes Navia \& Flechtmann, 2003a}

Type host: Attalea dubia (Mart.) Burret.

Type locality: Brazil (Pariquera-Açu, São Paulo)

Plant part: upper leaf surface

Remarks: seen as white wax stripes on the leaf surface (Navia \& Flechtmann 2003a).

\section{Notostrix flabelliferae Mohanasundaram, 1982}

Type host: Borassus flabellifer $\mathrm{L}$.

Type locality: India (Vriddhachalam, Tamil Nadu)

Plant part: midrib of unopen leaf

\section{Notostrix jamaicae Keifer, 1970}

Type host: Cocos nucifera $\mathrm{L}$.

Type locality: Jamaica (Woodstock)

Other host: Attalea sp.; Syagrus romanzoffiana (Cham.) Glassm. (Santana \& Flechtmann 1998)

Other locality: Brazil (Distrito Federal, Rio de Janeiro) (Santana \& Flechtmann 1998); Costa Rica (Schliesske 1988)

Plant part: leaf

\section{Notostrix longiseta Navia \& Flechtmann, 2003a}

Type host: Butia archeri (Glass.) Glass.

Type locality: Brazil (Pirassununga, São Paulo)

Plant part: upper leaf surface

Remarks: seen as white wax stripes on leaf surface (Navia \& Flechtmann 2003a).

Notostrix miniseta Navia \& Flechtmann, 2003a 
Type host: Bactris vulgaris Barb. Rodr.

Type locality: Pariquera-Açu, São Paulo, Brazil

Other host: Bactris setosa Mart.

Plant part: upper leaf surface

Remarks: seen as white wax stripes on leaf surface (Navia \& Flechtmann 2003).

Notostrix nasutiformes Gondim Jr., Flechtmann \& Moraes, 2000

Type host: Cocos nucifera L.

Type locality: Brazil (Igarassu, Pernambuco)

Other host: Attalea dubia (Mart.) Burret.; Attalea phalerata Mart. (this piblication); Bactris ferruginea Burret. ex Spreng.; Bactris gasipaes Kunth; Bactris setosa Mart.

Other locality: Brazil (São Paulo) (Gondim Jr. et. al. 2000); Mexico (Chetumal, Quintana Roo) (this publication)

Plant part: upper leaf surface

Notostrix spinula Navia \& Flechtmann, 2005

Type host: Mauritia flexuosa L.

Type locality: Brazil (Manaus, Amazonas)

Plant part: upper leaf surface

Notostrix trifida Navia \& Flechtmann, 2003a

Type host: Lytocaryum hoehnei (Burret.) Toledo

Type locality: Brazil (Cotia, São Paulo)

Plant part: upper leaf surface

Remarks: seen as white wax stripes on leaf surface (Navia \& Flechtmann 2003).

\section{Notostrix vazquezae Navia \& Flechtmann, 2003a}

Type host: Sabal sp.

Type locality: Mexico (Chetumal, Quintana Roo)

Plant part: upper leaf surface

\section{Phyllocoptinae, Calacarini}

\section{Calacarus palmae Mohanasundaram, 1994}

Type host: Collinia sp.

Type locality: India (Tamil Nadu)

\section{Phyllocoptinae, Phyllocoptini}

\section{Acritonotus denmarki Keifer, 1962c}

Type host: Roystonea elata (Bartr.) F. Harper

Type locality: USA (Fort Lauderdale, Florida)

Other host: Cocos nucifera L. (Moore \& Howard 1996)

Plant part: leaf

Remarks: type species of the genus (Amrine Jr. \& de Lillo 2003). It was observed causing leaf russeting 
on young plants (Keifer 1962c).

\section{Adenoptus chamaeropsi Mitrofanov, Sekerskaya \& Sharonov, 1983}

Type host: Chamaerops humilis L.

Type locality: Ukraine (Yalta, Crimea)

Remarks: type species of the genus (Amrine Jr. \& de Lillo 2003).

\section{Adenoptus migeoni Navia \& Flechtmann, 2003b}

Type host: Chamaerops humilis L.

Type locality: France (Montpellier, Languedoc-Roussillon)

Plant part: upper leaf surface

\section{Amrineus cocofolius Flechtmann, 1994}

Type host: Cocos nucifera L.

Type locality: Brazil (Jales, São Paulo)

Other host: Attalea geraensis Barb. Rodr.; Butia archeri (Glass.) Glass.; Butia eriospatha (Mart.) Becc.; Syagrus romanzoffiana (Cham.) Glassm. (this publication)

Other locality: Brazil (Bahia, Ceará, Pernambuco, Rio de Janeiro, Sergipe) (Flechtmann 1997; Santana \& Flechtmann 1998; Navia et al. 2005); Mexico (Yucatan) (Navia et al. 2005)

Plant part: leaf (Flechtmann, 1994); fruit (Ferreira et al. 2001; Navia et al. 2005)

Remarks: type species of the genus (Amrine Jr. \& de Lillo 2003). It was observed causing chlorotic spots that may coalesce (Flechtmann 1994). Redescribed by Flechtmann (1997).

\section{Amrineus coconuciferae (Keifer, 1962a)}

Acamina coconuciferae Keifer, 1962 (Flechtmann 1994)

Type host: Cocos nucifera $\mathrm{L}$.

Type locality: USA (Coral Gables, Florida)

Plant part: leaf

\section{Calepitrimerus livistonae Wei \& Feng, 2002}

Type host: Livistona chinensis (Jacq.) R. Barb.

Type locality: China (Nanning, Guangxi Zhuang)

\section{Epitrimerus calami Keifer, 1969}

Type host: Calamus australis Mart.

Type locality: Australia (Nambour, Queensland)

Plant part: leaf

Remarks: it was observed causing yellowish or brownish spots of 0.5 to $1 \mathrm{~cm}$ in diameter on the upper leaf surface. Seen as white wax flocks on leaf surface.

\section{Epitrimerus elaeis Boczek \& Natcheff, 1989}

Type host: Elaeis guineensis Jacquin

Type locality: Côte d'Ivoire (Ivory Coast) (Lame)

Plant part: under leaf surface

Remarks: it was observed causing some discoloration.

Epitrimerus englerus Huang, 2001 
Type host: Arenga englera Beccari

Type locality: Taiwan (Hualien, Walapi)

Plant part: upper leaf surface

\section{Epitrimerus steveni Mitrofanov, Sekerskaya \& Sharonov, 1983}

Type host: Chamaerops humilis L.

Type locality: Ukraine (Yalta, Crimea)

Plant part: leaf

\section{Gilarovella caniculata Mitrofanov, Sekerskaya \& Sharonov, 1983}

Type host: Chamaerops humilis L.

Type locality: Ukraine (Yalta, Crimea)

Plant part: leaf

Remarks: type species of this monospecific genus (Amrine Jr. \& de Lillo 2003).

\section{Neocupacarus flabelliferis Das \& Chakrabarti, 1985}

Type host: Borassus flabellifer $\mathrm{L}$.

Type locality: India (Massanjore, Santhalparganas, Bihar)

Plant part: leaf

Remarks: type species of this monospecific genus (Amrine Jr. \& de Lillo 2003).

\section{Phyllocoptes mariaui Boczek \& Natcheff, 1989}

Type host: Elaeis guineensis Jacquin

Type locality: Côte d’Ivoire (Ivory Coast) (Lame)

Plant part: under leaf surface

Remarks: colonies are found under a thin wax layer that occurs naturally on the leaf surface of the host plant, and may cause some discoloration.

\section{Euterpia fissa Navia \& Flechtmann, 2005}

Type host: Euterpe precatoria Mart.

Type locality: Brazil (Manaus, Amazonas)

Plant part: leaf upper surface

Remarks: type species of this monospecific genus (Amrine Jr. \& de Lillo 2003).

\section{Phyllocoptinae, Tegonotini}

\section{Glabrisceles euterpis Navia \& Flechtmann, 2002}

Type host: Euterpe edulis Mart.

Type locality: Brazil (Pariquera-Açu, São Paulo)

Plant part: terminal shoot

Remarks: type species of this monospecific genus (Amrine Jr. \& de Lillo 2003).

\section{Scolocenus spiniferus Keifer, 1962c}

Type host: Cocos nucifera $\mathrm{L}$.

Type locality: Philippines (Guinobatan, Albay)

Plant part: leaf 
Remarks: type species of this monospecific genus (Amrine Jr. \& de Lillo 2003). Its possible action as vector of the virus that causes the coconut disease known as "cadang-cadang" in Philippines was investigated, with negative results (Briones \& Sill Jr. 1963).

\section{Tegonotus gutierrezi Boczek \& Natcheff, 1989}

Type host: Elaeis guineensis Jaquin

Type locality: Côte d’Ivoire (Ivory Coast) (Lame)

Plant part: leaf under surface

Remarks: it was observed causing some discoloration (Boczek \& Natcheff 1989).

\section{Phytoptidae}

\section{Phytoptinae}

\section{Acathrix lobata Navia \& Flechtmann, 2002}

Type host: Geonoma brevispatha Barb. Rodr.

Type locality: Brazil (São Pedro, São Paulo)

Other host: Geonoma schottiana Mart.

Plant part: leaf; terminal shoot

\section{Acathrix trymatus Keifer, 1962b}

Type host: Cocos nucifera L.

Type locality: Philippines (Guinobatan, Albay)

Other locality: USA (Florida) (Welbourn $1997^{1}$ in Amrine Jr. \& de Lillo 2003).

Plant part: terminal shoot

Remarks: type species of the genus (Amrine Jr. \& de Lillo 2003). Found in large numbers in terminal shoots of $C$. nucifera affected by "cadang-cadang" disease. However, its possible action as vector of the virus that causes the coconut disease known as "cadang-cadang" in the Philippines was investigated, with negative results (Briones \& Sill Jr. 1963).

\section{Sierraphytoptinae, Mackiellini}

\section{Mackiella borasis Mohanasundaram, 1981}

Type host: Borassus flabellifer $\mathrm{L}$.

Type locality: India (Vriddhachalam, Tamil Nadu)

Plant part: fold of unopened leaf

\section{Mackiella phoenicis Keifer, 1939a}

Type host: Phoenix dactylifera L.

Type locality: USA (Riverside, California)

Plant part: unopened young leaf

Other localitiy: Iraq (Baghdad) (Mohamed \& El-Haidari 1968)

Remarks: type species of the genus (Amrine Jr. \& de Lillo 2003).

\section{Palmiphytoptus barbosae Navia \& Flechtmann, 2005}

1. Welbourn, C. (1997) Acathrix trymatus Keifer in Florida. 
Type host: Astrocaryum acaule Mart.

Type locality: Brazil (Manaus, Amazonas)

Plant part: upper leaf surface

Remarks: colonies were found under a dense trichoma layer along the midrib on the upper leaf surface.

\section{Palmiphytoptus oculatus Navia \& Flechtmann, 2002}

Type host: Bactris setosa Mart.

Type locality: Brazil (Cananéia, São Paulo)

Other host: Astrocaryum aculeatissimum (Schott) Burret (Navia \& Flechtmann 2002)

Plant part: terminal shoot; leaf (Navia \& Flechtmann 2002)

Remarks: type species of the genus (Amrine Jr. \& de Lillo 2003).

\section{Propilus alternatus Navia \& Flechtmann, 2005}

Type host: Mauritia flexuosa $\mathrm{L}$.

Type locality: Brazil (Manaus, Amazonas)

Plant part: upper leaf surface

\section{Propilus gentyi Keifer, 1975b}

Type host: Aiphanes sp.

Type locality: Colombia (Bucaramanga, Santander)

Other host: Astrocaryum aculeatissimum (Schott) Burret; Bactris vulgaris Barb. Rodr. (this publication)

Other locality: Brazil (São Paulo) (this publication)

Plant part: upper leaf surface

Remarks: type species of the genus (Amrine Jr. \& de Lillo 2003). It was observed causing russeting of the affected tissue.

\section{Propilus pellitus Navia \& Flechtmann, 2002}

Type host: Lytocaryum hoehnei (Burret.) Toledo

Type locality: Brazil (Cotia, São Paulo)

Plant part: upper leaf surface

\section{Propilus spinosus Keifer, 1975b}

Type host: Aiphanes sp.

Type locality: Colombia (Bucaramanga, Santander)

Other host: Bactris setosa Mart.; Syagrus romanzoffiana (Cham.) Glassm. (this publication)

Other locality: Brazil (São Paulo) (this publication)

Plant part: upper leaf surface

Remarks: type species of the genus (Amrine Jr. \& de Lillo 2003). It was observed causing russeting of the affected tissue.

\section{Propilus syagris Gondim Jr., Flechtmann \& Moraes, 2000}

Type host: Syagrus romanzoffiana (Cham.) Glassm.

Type locality: Brazil (Pariquera-Açu, São Paulo)

Other host: Astrocaryum aculeatissimum (Schott) Burret; Bactris vulgaris Barb. Rodr.

(this publication)

Other locality: Brazil (Rio de Janeiro) (this publication) 
Plant part: terminal shoot; upper leaf surface

\section{Propilus tavaresi Navia \& Flechtmann, 2005}

Type host: Elaeis oleifera (Kunth) Cortés

Type locality: Brazil (Manaus, Amazonas)

Plant part: upper leaf surface

Remarks: described from specimens collected under a dense trichoma layer along the midrib on the upper leaf surface of E. oleifera.

\section{Retracrus elaeis Keifer, 1975a}

Type host: Elaeis guineensis Jacquin

Type locality: Colombia (San Alberto, Bucaramanga)

Other host:; Bactris gasipaes Kunth (Ochoa et al. 1994); Chamaedorea costaricana Oerst. (this publication); Cocos nucifera L. (Genty \& Reyes 1977)

Other locality: Costa Rica (Salas ${ }^{1}$, personal communication cited by Schlieske 1988); Brazil (São Paulo) (this publication)

Plant part: leaf

Remarks: it was observed causing numerous spots on leaves, initially dark, gradually becoming yellowish or orange. Some infested leaves can dry completely. Attacked plants can have yield impaired.

\section{Retracrus johnstoni Keifer, 1965}

Type host: Chamaedorea sp.

Type locality: Mexico intercepted at USA (San Antonio, Texas)

Other host: Astrocaryum aculeatissimum (Schott) Burret; Bactris gasipaes Kunth; Bactris setosa Mart. (this publication); Chamaedorea costaricana Oerst. (Ochoa et al. 1994); Chamaedorea elegans Mart. (Schliesske 1988); Cocos nucifera L. (Santana et al. 1994); Elaeis guineensis Jacquin; Euterpe edulis Mart.; Euterpe oleracea Mart. ; Euterpe precatoria Mart. ; Euterpe sp. ; Geonoma gamiovora Barb. Rodr.; Geonoma pohliana Mart.; Geonoma schottiana Mart.; Mauritia flexuosa L.; Scheelea sp. (this publication); and Syagrus romanzoffiana (Cham.) Glassm. (Santana \& Flechtmann 1998)

Other locality: Brazil (Amazonas, Sergipe, São Paulo) (Santana et al. 1994; this publication); Costa Rica (Schliesske 1988)

Plant part: upper leaf surface

Remarks: type species of the genus (Amrine Jr. \& de Lillo 2003). Found in dense colonies, causing spots that are dark when observing the lower surface, but yellowish when observing the upper surface.

\section{A key to Eriophyoidea associated with palm trees worldwide}

1 External vertical setae (ve) present; internal vertical seta (vi) absent. Gnathosoma often large, but with chelicerae straight or slightly and evenly curved Phytoptidae Murray ... 3

- Prodorsal shield without vi or ve. Gnathosoma variable . 2

2 Gnathosoma usually small in relation to idiosoma; when large, with chelicerae straight or slightly curved. Female genital epigynium usually with longitudinal ridges. Tibiae not fused with tarsi; tibial seta $(l)$ present; prodorsal shield not reduced, with tubercles...

Eriophyidae Nalepa ... 16

- Gnathosoma large in relation to idiosoma; chelicerae abruptly curved near base and bent down. Female

1. Salas, L.A. Laboratorio de Acarologia e Nematologia, .... Universidade de Costa Rica. 
genital epigynium usually smooth. Legs with six segments, tibia not fused to tarsus; all coxal setae present; empodium divided.

Diptilomiopidae Keifer ... 59

3 Body vermiform. Opisthosomal annuli narrow and subequal dorsoventrally. Opisthosomal setae $c 1$ present

Phytoptinae Murray

Femur and genua not fused. Scapular setae $(s c)$ and tubercles minute. Prodorsal shield with medio-posterior gland. Acathrix Keifer ... 4

- Body fusiform and often flattened. Opisthosomal annuli usually broad, with dorso-ventral differentiation ... Sierraphytoptinae Keifer. Opisthosomal setae $c 1$ absent Mackiellini Keifer ... 5

4 Sinuous longitudinal lines over whole prodorsal shield. Frontal lobe absent. Empodium with 11-12- rays Acathrix trymatus Keifer

- Sinuous longitudinal lines restricted to posterior part of prodorsal shield; anterocentral area smooth. Frontal lobe present. Empodium with 9-rays Acathrix lobata Navia \& Flechtmann

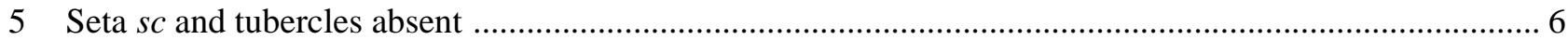

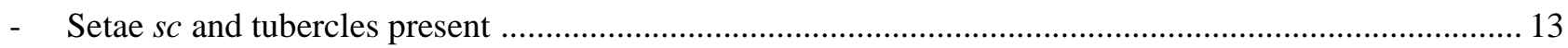

6 Dorsal annuli much broader than ventral annuli Propilus Keifer ... 7 Opisthosomal annuli subequal dorsoventrally Palmiphytoptus Navia \& Flechtmann ... 12

7 Lateral projections of dorso-opisthosomal annuli well-developed, spine-like or in a pattern of alternating shorter and longer projections

- Lateral projections of dorso-opisthosomal annuli moderate, not spine-like

8 Lateral projections of dorsal opisthosomal annuli in a pattern of alternate shorter and longer projections . Propilus alternatus Navia \& Flechtmann

- Lateral projections of dorsal opisthosomal annuli spine-like 9

9 Seta ve visible in dorsal view, on humeral angles of prodorsal shield. Epigynium smooth. Frontal lobe prominent, acuminate Propilus spinosus Keifer

- Seta ve not visible in dorsal view, on the ventrally bent anterior part of prodorsal shield. Epigynium with longitudinal lines. Frontal lobe short, apically rounded

Propilus syagris Gondim Jr., Flechtmann \& Moraes

10 Dorsal opisthosomal annuli microtuberculate. Seta ve slightly behind anterolateral shield margin

Propilus tavaresi Navia \& Flechtmann

Dorsal opisthosomal annuli smooth. Setae ve on or slightly anterior to anterolateral shield margin ....... 11

11 Frontal lobe subtriangular and apically acuminate. Epigynium basally covered by a delicate, transversally striate membrane. Empodium with 9 rays

Propilus pellitus Navia \& Flechtmann

- Frontal lobe short and apically rounded. Epigynium basally not covered by a membrane. Empodium with 3 rays

Propilus gentyi Keifer

12 Prodorsal shield with median and admedian lines on central shield; eye-like structures absent. Empodium with 12 rays. Dorsal-opisthosoma annuli smooth Palmiphytoptus barbosae Navia \& Flechtmann

- Prodorsal shield with central area not ornamented; eye-like structure present. Empodium with 8 rays. Dorsal-opisthosoma annuli microtuberculate Palmiphytoptus oculatus Navia \& Flechtmann

13 Setae $s c$ and $v e$ on enlarged tubercles, bulbous, directing ve forward and $s c$ backward

Retracrus Keifer ... 14

Setae sc and ve on normal tubercles, directing ve and sc forward Mackiella Keifer ... 15

14 Epigynium ornamented with a transverse row of short basal longitudinal lines. Prodorsal shield with faint longitudinal lines

Retracrus elaeis Keifer

- Epigynium smooth without a transverse row of short basal longitudinal lines. Prodorsal shield without longitudinal lines

Retracrus johnstoni Keifer

15 Opisthosoma with dorso-ventral differentiation; wide tergites with elongated microtubercles, like longitudinal lines. Empodium with 7 rays Mackiella phoenicis Keifer 
- Ospisthosoma subequal dorso-ventrally; laterally continuous annulus and uniformly microtuberculated. Empodium with 6 rays

Mackiella borasis Mohanasundaram

16 Epigynium apressed to coxae, separating coxae more than normal and in lateral view projecting from venter, with ridges in 2 uneven ranks. Sternal line shortened ... Cecidophyinae Keifer. Seta $s c$ and tubercles present ...Colomerini Newkirk \& Keifer. Body vermiform. Prodorsal shield without frontal lobe over gnathosoma. Seta $s c$ directed forward, centrally or laterally, but not backward. All normal leg and opisthosoma setae present ...Colomerus Newkirk \& Keifer. Dorsal tubercles well anterior to rear shield. Ocellar spot on lateral shield. Empodium with 7 rays.

Colomerus novahebridensis Keifer

- Epigynium not apressed to coxae or separating them and in lateral view not projecting from venter, with variable ornamentation, without ridges in 2 ranks. Sternal line not shortened...

17 Body vermiform; annuli subequal dorso-ventrally, at least on anterior half to two-thirds of opisthosoma. Prodorsal shield usually lacking a frontal lobe, or with a slight projection over gnathosoma base. If frontal lobe is present over gnathosoma, then the lobe is narrow, basally flexible, and combined with narrow opisthosomal annuli

Eriophyinae Nalepa ... 18

- Body usually fusiform. Opisthosoma with broad and smooth dorsal annuli and narrow and microbuberculated ventral annuli. Prodorsal shield usually with a broad-based and rigid frontal lobe over gnathosoma. If frontal lobe is not present or if it is too small, annuli differ dorso-ventrally at least in larger dorsal microtubercles. If annuli are subequal dorso-ventrally and a broad-based frontal lobe is absent, then annuli are as broad as the epigynium

Phyllocoptinae Nalepa ... 23

18 Seta $s c$ on, or very near, rear margin, with transverse basal axis, directed backward, usually divergently .. Aceriini Amrine \& Stasny. Tibial seta I ( $l$ ) present. Coxal seta $l b$ present. Dorsal opisthosoma evenly arched in transversal section. Posterior opisthosoma with continuous and dorsoventrally subequal annuli. Some species with a frontal lobe small and with a flexible base Aceria Keifer ... 19

- Seta $s c$ more or less anterior to rear margin, directed forward or upward. If $s c$ is near shield margin, the seta is directed forward or has longitudinal basal axis Eriophyini Nalepa ... 22

19 Prodorsal shield with longitudinal lines over whole central area 20

- Prodorsal shield without longitudinal lines on central area 21

20 Epigynium with granules on anterior region. Empodium with 8 lateral, paired rays and one apical, unpaired ray Aceria biornata Navia \& Flechtmann

- Epigynium without granules on anterior region. Empodium with 6 lateral, paired rays

Aceria guerreronis Keifer

21 Legs robust, opisthosoma slightly fusiform, resembling Cisaberoptus Keifer protogyne. Anterior region of epigynium with transversal microtuberculated lines. Frontal lobe absent

Aceria translinea Navia \& Flechtmann

- Legs normally developed, opisthosoma typically vermiform. Epigynium with longitudinal lines. Frontal lobe subtriangular, with basal line flexible Aceria gymnoscuta Navia \& Flechtmann

22 Coxal seta $l b$ absent. Seta $s c$ directed anterolaterally and on tubercles with longitudinal basal axis. Frontal lobe small. Genual seta I ( $l$ ) shorter than leg I ... Nacerimina Keifer. Coxi-genital region ornamented with numerous short dashes arranged longitudinally. Epigynium with about 14 longitudinal lines. Empodium with 7 rays

Nacerimina gutierrezi Keifer

- Coxal seta $1 b$ present. Base of scapular setae (sc) tubercles variable. Frontal lobe large, triangular, with straight or concave lateral margins. Genual seta I $\left(l^{\prime}\right)$ enlarged and longer than leg I ...Proartacris Mohanasundaram. Body elongate and attenuate. Absence of microtubercles on dorso-opisthosomal annuli .......

Proartacris longior Navia \& Flechtmann

23 Empodium divided Acaricalini Amrine \& Stasny ... 24

- Empodium undivided 29

24 Seta $s c$ absent. No setae of tibia I $\left(l^{\prime}\right)$, genu II $\left(l^{\prime}\right)$, femur $(b v)$ and ventral-opisthosomal I $(d)$ and II $(e)$.. 


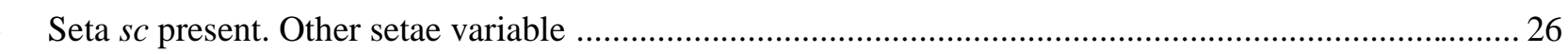

25 Opisthosoma with a central, longitudinal ridge extending over at least the anterior $3 / 4$ of opisthosoma. In dorsal view, dorsal annuli project laterally in an uneven series

Knorella Keifer. Triangular area laterad of coxisternum II with a tuberculate pattern. Epigynium with longitudinal striae restricted to basal area. Opisthosoma with lateral projections on annuli 2, 4, 7, 10, 12 and 15 Knorella geonomae Gondim Jr., Flechtmann \& Moraes

- Opisthosoma with a central, longitudinal ridge extending over 4 anterior annuli, followed by a wide median furrow. In dorsal view, dorsal annuli project in an even series ... Schizacea Keifer. Empodium bipartite, with 7-8 rays in each branch. Five smooth coxi-genital annuli

Schizacea geonomae Navia \& Flechtmann

26 Opisthosoma with several dorsal annuli fused into a plate immediately posterior to prodorsal shield. Opisthosomal ventral seta I (d) present. Seta $s c$ spine-like ... Nasuchus Navia \& Flechtmann. Only species in the genus

Nasuchus pindobates Navia \& Flechtmann

- Opisthosoma with several dorsal annuli fused into a plate not immediately posterior to prodorsal shield, first three opisthosomal annuli narrow, followed by about 10 fused annuli as wide as shield. Seta $d$ absent. Seta $s c$ not spine-like Tumescoptes Keifer ... 27

27 No ridges or furrows on posterior dorsal-opisthosoma (posterior to plate of fused annuli)

Tumescoptes phoenixi Meyer

With ridges or furrows on posterior dorsal-opisthosoma (posterior to plate of fused annuli). 28

28 Posterior dorsal-opisthosoma (posterior to plate of fused annuli) with one median and two lateral furrows. Leg unguinal seta $(u)$ bifurcate Tumescoptes dicrus Meyer

- Posterior dorsal-opisthosoma (posterior to plate of fused annuli) with one median ridge. Seta $u^{\prime}$ not bifurcate

Tumescoptes trachycarpi Keifer

29 Seta $s c$ absent. Tubercles present or absent. Opisthosomal annuli, in dorsal view, without lateral projections, lobes or a plate posterior to prodorsal shield with lateral extensions ... Calacarini Amrine \& Stasny ...Opisthosoma with three dorsal ridges. Median ridge narrow, as long as lateral ridges. Tibial seta I ( $(l)$ present. Opisthosoma gradually attenuate...Calacarus Keifer ... Only species in the genus associated with palm trees...... Calacarus palmae Mohanasundaram

- Seta $s c$ present. Other characters variable 30

30 Opisthosoma, in dorsal view, with lateral lobes or pointed projections from some annuli, or with a plate posterior to prodorsal shield bearing lateral extensions Tegonotini Bagdasarian ... 31

- Opisthosoma, in dorsal view, without lateral lobes, pointed projections from some annuli or a plate posterior to prodorsal shield bearing lateral extensions

31 Anterior dorsal-opisthosoma annuli not fused. Prodorsal shield without posterior projections over opisthosoma. Opisthosomal annuli not spine-like. Prodorsal shield not separated from opisthosoma by a transversal depression. Seta $s c$ inserted anterior to rear shield margin. Frontal lobe not emarginate. Coxal seta $1 b$ and tibial seta $l$ ' present ...Tegonotus Nalepa. Empodium with 7 rays. Epigynium with short lines irregularly distributed. Seta $s c$ directed centrad Tegonotus gutierrezi Boczek \& Natcheff

- Anterior dorsal-opisthosoma annuli fused. Other characters variable 32

32 Dorsal-opisthosomal plate (annuli fused) without spine-like projections ...Glabrisceles Navia \& Flechtmann. Only species in the genus.

Glabrisceles euterpis Navia \& Flechtmann

- Dorsal-opisthosomal plate (annuli fused) with three spine-like projections...Scolocenus Keifer. Only species in the genus.

Scolocenus spiniferus Keifer

33 Tubercle of $s c$ on or very near rear shield margin, directing seta backward, usually divergently; tubercle subcylindrical, with transversal base ... Anthocoptini Amrine \& Stasny. Dorsal-opisthosoma with a wide median furrow. Prodorsal shield without posterior projection. Coxal seta $l b$ present. Genual seta II $\left(l^{\prime}\right)$ 
absent

- Tubercle of $s c$ anterior to rear shield margin, directing seta backward, upward or convergently; if tubercle and seta are near shield margin, then tubercle is subcylindrical and have longitudinal or diagonal base ... Phyllocoptini Nalepa 46

34 Epigynium with two transversal rows of longitudinal lines or ribs Notostrix exigua Flechtmann

- Epigynium with a single row of longitudinal lines which can occupy the whole epigynium or the basal region can be granulated or have short or broken lines 35

35 Seta $s c$ short $(\leq 8 \mu \mathrm{m}$ long $)$, not reaching shield rear margin 36

- $\quad$ Seta $s c$ long (> $8 \mu \mathrm{m}$ long), usually reaching or extending beyond shield rear margin 41

36 Empodium divided, bipartite. In leg I, tibial $l^{\prime}$ longer than or as long as lateral seta $\left(f t^{\prime}\right)$

Notostrix fissipes Navia \& Flechtmann

- Empodium undivided or tripartite. In leg I, seta $l^{\prime}$ variable in length

37 Empodium tripartite. Epigynium basally granulated, with centrally convergent longitudinal lines. Seta $s c$ minute $(2 \mu \mathrm{m}$ long) which can be covered with wax

Notostrix miniseta Navia \& Flechtmann

- $\quad$ Empodium undivided. Epigynium variable. Seta sc 2 to $8 \mu \mathrm{m}$ long, not covered by wax....................... 38

38 Epigynium with a basal pattern of short curved lines, somewhat separated from the row of 18-20 longitudinal lines or ribs

Notostrix jamaicae Keifer

- Epigynium with basal granules or longitudinal lines somewhat broken anteriorly 39

39 Epigynium with a row of less than 15 longitudinal lines; with granulate pattern on anterior half.

Notostrix nasutiformes Gondim Jr., Flechtmann \& Moraes

- Epigynium with a row of more than 15 longitudinal lines; if anterior region with granules, they occupy just basal portion (not anterior half)

40 Epigynium with a row of 15-19 longitudinal lines, broken anteriorly and covering the whole epigynium .

Notostrix butiae Gondim Jr., Flechtmann \& Moraes

- Epigynium with a row of 24 (20-24) longitudinal lines, some of which are anteriorly or posteriorly joined by curved lines; anteriorly granulated Notostrix spinula Navia \& Flechtmann

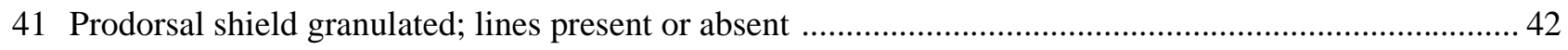

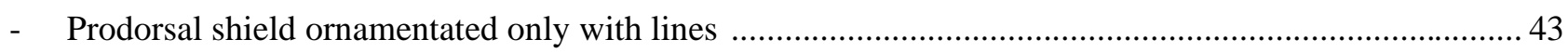

42 Prodorsal shield anteriorly rounded, totally covered with rounded granules

Notostrix flabelliferae Mohanasundaram

- Prodorsal shield anteriorly pointed, with irregular granules, in some areas elongate, and covered by wax. Ornamentation restricted to the central region delimited by the submarginal line

Notostrix acuminata Navia \& Flechtmann

43 Empodium tripartite, with two lateral rayed branches and a central branch apically bifurcated. Femoral seta $(b v)$ angulated

Notostrix trifida Navia \& Flechtmann

- Empodium undivided. Femoral seta $(b v)$ straight ..... 44

44 Epigynium basally granulated. Seta $s c$ reaching or extending over $3^{\text {rd }}-4^{\text {th }}$ dorsal annulus

Notostrix longiseta Navia \& Flechtmann

- $\quad$ Epigynium without granules. Seta $s c$ not reaching $3^{\text {rd }}-4^{\text {th }}$ dorsal annulus 45

45 Epigynium with longitudinal lines or ribs distinctively divergent basally. Female coxal region with curved lines, whithout granules Notostrix attenuata Keifer

- Epigynium with longitudinal lines not divergent anteriorly. Female coxal region granulated and with faint short lines

Notostrix vazquezae Navia \& Flechtmann

46 Prodorsal shield with a long and narrow posterior projection, extending posteriorly over opisthosoma for 8 annuli, slightly curved to one of the sides. Dorsal opisthosoma with a median furrow flanked by wider submedian furrows. Tibial seta I ( $l$ ) and coxal seta $1 b$ present...Gilarovella Mitrofanov, Sekerskaya \& Sharonov. Only species in the genus Gilarovella caniculata Mitrofanov, Sekerskaya \& Sharonov 
- Prodorsal shield without projections. Other characters variable 47

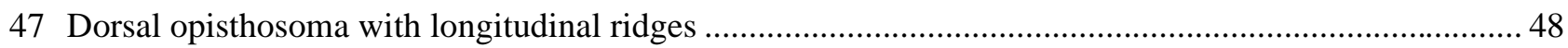

- Dorsal opisthosoma without longitudinal ridges ..................................................................55

48 Dorsal opisthosoma with one longitudinal ridge. Antapical seta $(d)$ bifurcate. Absence of opisthosomal ventral seta I $(d)$ and II $(e)$, femoral setae $(b v)$ I and II. Presence of tibial I $\left(l^{\prime}\right)$ and genual setae I and II $\left(l^{\prime}\right)$... Euterpia Navia \& Flechtmann. Only species in the genus ..... Euterpia fissa Navia \& Flechtmann

- Dorsal opisthosoma with 3 longitudinal ridges. Other characters variable .......................................49

49 Femoral setae $(b v)$, opisthosomal lateral $(c 2)$ and ventral II $(e)$ absent. Epigynium with short lines; longitudinally divided by shaping a "rounded w". Solenidium short and knobbed Amrineus Flechtmann ... 50

- Femoral $(b v)$ and all opisthosomal setae present. Other characters variable ...................................... 51

50 Epigynium with long longitudinal lines. Five coxi-genital annuli ........ Amrineus coconuciferae (Keifer)

- Epigynium without long longitudinal lines, only with dispersed short lines. Eight to nine coxi-genital annuli

Amrineus cocofolius Flechtmann

51 Median opisthosomal ridge ending posteriorly in broad furrow before the end of the submedian ridges ... Calepitrimerus Keifer. Seta sc convergent. Epigynium with 2 transversal rows of longitudinal lines. Empodium with 4 rays.

Calepitrimerus livistonae Wei \& Feng

- Median opisthosomal ridge fading at about same level as submedian ridges. Shallow submedian furrows producing low median ridge

Epitrimerus Nalepa ... 52

52 Prodorsal shield ornamented with many short lines; admedian lines extending from rear margin to $1 / 3$ anterior shield, apically bifurcate

Epitrimerus englerus Huang

- Prodorsal shield not ornamented with short lines. Other characters variable 53

53 Prodorsal shield without posterior median extension. Empodium with more than 4 rays

Epitrimerus elaeis Boczek \& Natcheff

- $\quad$ Prodorsal shield with posterior median extension interrupting first dorsal annuli or arching then medially. Empodium with 4 or less rays 54

54 Epigynium with longitudinal lines. Empodium with 4 rays Epitrimerus calami Keifer

- Epigynium without longitudinal lines. Empodium with 2 rays Epitrimerus steveni Mitrofanov, Sekerskaya \& Sharonov

55 Dorsal opisthosoma with longitudinal furrows ................................................................. 56

- Dorsal opisthosoma without longitudinal furrows ..................................................................... 58

56 Dorsal opisthosoma with median and submedian longitudinal furrows. Opisthosomal ventral setae I (d) and II (e) absent ... Neocupacarus Das \& Chakrabarti. Only species in the genus

Neocupacarus flabelliferis Das \& Chakrabarti

- Dorsal opisthosoma with a median furrow flanked by ridges thickened for wax secretion. Opisthosomal ventral setae I $(d)$ and II $(e)$ present

Adenoptus Mitrofanov, Sekerskaya \& Sharanov ... 57

57 Seta $d$ bifurcated. First 5-6 dorsal opisthosomal annuli without median furrows and evenly microtuberculate; median furrow beginning posterior to these annuli. Epigynium with irregular texture, seemingly porous. Empodium with 3 rays

Adenoptus migeoni Navia \& Flechtmann

- Seta $d$ apparently not bifurcated. Median furrow of opisthosoma beginning immediately posterior to rear margin of prodorsal shield; no microtubercles on median furrow. Epigynium smooth. Empodium with 2 rays. Adenoptus chamaeropsi Mitrofanov, Sekerskaya \& Sharonov

58 Opisthosoma with dorsal annuli disorganized in the central region ... Acritonotus Keifer. Prodorsal shield not ornamented. No accessory seta. Empodium with 3 rays .... Acritonotus denmarki Keifer

- Opisthosoma with dorsal annuli organized in the central region ...Phyllocoptes Nalepa. Prodorsal shield with some lateral lines. Distal region of epigynium with concave, transversal lines, divided in 2 rows; proximal region with irregular, oblique and convergent lines. Empodium with 3 rays, palmed.....

Phyllocoptes mariaui Boczek \& Natcheff 
59 Seta $s c$ absent; tubercles present or absent. Tibia I ( $l$ ) and genu II $\left(l^{\prime}\right)$ setae present or absent 60

- Seta $s c$ and tubercles present. Tibia I $(l)$ and genu II $\left(l^{\prime \prime}\right)$ setae present

60 Seta $c_{2}$ absent. Opisthosoma without longitudinal ridges. Tibia I $(l)$ and genu II $\left(l^{\prime}\right)$ setae absent ... Davisella Flechtmann, Amrine \& Stasny ... Prodorsal shield smooth, with two circles in central region, next to anterior margin. Epigynium wider than distance between external angles of coxae II.....

Davisella palmea (Flechtmann)

- Seta $c_{2}$ present. Opisthosoma with 5 wax-bearing ridges. Tibia I $(l)$ and genu II ( $\left.l^{\prime}\right)$ setae present ... Neodialox Mohanasundaram. Only species in the genus

Neodialox palmyrae Mohanasundaram

61 Frontal lobe emarginated anteriorly. Prodorsal shield with 2 prominent transversal grooves, one near rear shield margin and another on rear shield margin. Tibia distinctly long ... Dialox Keifer. Only species in the genus.

Dialox stellatus Keifer 1962

- Frontal lobe not emarginated. Prodorsal shield without transversal grooves on posterior region. Tibia not distinctly long. ... Diptacus Keifer ...Empodium bipartite, each branch with 3 rays

Diptacus borinquensis Cromroy

\section{Discussion}

Palm trees are widely used for different reasons. Many species are cultivated around the world in open fields or in protected environments as ornamentals. Others are important in tropical and subtropical areas for providing material used as food and drinks; especially important in this sense are the oil palm (E. guineensis) and coconut palm (C. nucifera).

Despite the significant economic importance of some eriophyoid species on palm trees, knowledge about the mites on them is relatively sparse, although a significant number of studies have been conducted recently on one of the mites (A. guerreronis) on coconut, to which it causes major damage. The latter crop provides staple food for people of different countries, especially in Southeast Asia, where it is one of the main crops.

Eriophyoid mites have been reported from only 50 of the 3500 known palm species. In recent surveys for eriophyoid mites on plants of that group in natural vegetation of different parts of Brazil, at least one species was found on each of the majority of the palm species evaluated in this study and other studies (Flechtmann $\&$ Santana 1997; Flechtmann 1989, 1994, 1998; Gondim Jr. et al. 2000; Navia \& Flechtmann 2002, 2003a; Robbs \& Perachi 1965; Santana \& Flechtmann 1998; Santana et al. 1994). Considering the socio-economic importance of palm trees around the world, and the potential pest status of many eriophyid mites, detailed studies of these mites on palms seem extremely desirable.

Four eriophyoid species were reported on palm trees in Europe, 6 in Africa, 17 in Asia, Pacific Islands and Australia, and 40 in the Americas. Four of the species reported belong to Diptilomiopidae, 44 to Eriophyidae and 14 to Phytoptidae.

Diversity of palm trees is extremely limited in Europe (Jones 1995). This might explain why only 4 eriophyoid species are known on palm trees from that continent, all on $C$. humilis. Yet, in Africa, where a high number of palm species and genera are known (Jones 1995), the number of eriophyoid species reported on palm trees is also small. Although this could be due to a naturally reduced number of these mites on palms on that continent, it seems that the real cause is the relatively low number of studies conducted.

By comparison, a relatively high number of eriophyoid species has been reported from the Americas, especially on palm trees from Brazil where this group of plants is very diverse. The relatively high number of eriophyoids in this case is mostly due to surveys conducted in the last fifteen years (Santana et al. 1994; Flechtmann \& Santana 1997; Flechtmann 1989, 1994, 1998; Santana \& Flechtmann 1998; Gondim Jr. et al. 2000; Navia \& Flechtmann 2002, 2003a, 2005).

Worldwide, the highest number of eriophyoid species on palm trees has been reported on coconut. Fifteen 
species have been found on this crop in the Americas, Southeast Asia, Africa and Oceania. Such a high number might be due to the wide distribution of the crop and to the more intense effort directed to the knowledge of pests on it. Most eriophyoid species associated with coconut are restricted to a geographic region, except $A$. guerreronis, which has been reported from America, Africa and recently from Southeast Asia.

Eriophyoids are usually quite host specific; most species have been reported from a single host or from hosts of the same genus (Oldfield 1996). Of the eriophyoid species associated with palm trees, 19 are reported from more than one host species. Seventeen of these eriophyoid species were reported from hosts belonging to different genera. The extreme case was $R$. johnstoni, collected from 18 palm species of 10 genera. Other cases are $N$. butiae on 6 palm species of 3 genera; $N$. nasutiformes on 5 palm species of 3 genera; A. cocofolius on 5 palm species of 4 genera; and A. guerreronis on 4 palm species of 4 genera. These results show that several eriophyoid species associated with palm trees have low host specificity, as reported for a few other species. However, it is often difficult to determine whether a certain degree of morphological variation indicates the existence of different species or just intra-specific variation. Thus, it is conceivable that at least in some cases complexes of very similar species are being identified under a single name. The use of modern tools, especially of molecular techniques, may be very useful in future studies for the identification of mites on palm trees, as well as on other plant groups.

\section{Acknowledgements}

To C.H.W. Flechtmann, for the invaluable collaboration in helping the identification of the mites reported here. To Dr. James Amrine Jr., for kindly provide updated database on Eriophyoid mites. To L.V.F. Silva, G.P. Arruda Filho, M.M. Vásquez, R.Q. Barbosa, A.M. Tavares and H. Aguilar, for the considerable time they spent helping in the collection of many of the species reported here. To CNPq for the PhD scholarship granted to the first author. To FAPESP (Fundação de Amparo a Pesquisa do Estado de São Paulo) for the partial support to this project within the BIOTA/FAPESP (Biodiversity Virtual Institute Program - www.biotasp.org.br).

This work was conducted at ESALQ-USP, Piracicaba, SP, Brazil, as a part of a thesis submitted by the senior author in partial fulfillment of the requirements for the Ph.D. degree.

\section{References}

Amrine Jr., J.W. \& de Lillo, E. (2003) A database on Eriophyoidea of the world. West Virginia University, Michigan (File Maker 4.0) (unpublished).

Amrine Jr., J.W. \& Manson, D.C.M. (1996) Preparation, mounting and descriptive study of Eriophyoid mites. In: Lindquist, E.E.; Sabelis, M.W. \& J. Bruin (Org.). Eriophyoid mites: their biology, natural enemies and control. Elsevier, Amsterdam, 383-396.

Amrine Jr., J.W. \& Stasny, T.A.H. (1994) Catalog of the Eriophyoidea (Acarina: Prostigmata) of the world. Indira Publishing House, West Bloomfield, 798pp.

Amrine Jr., J.W., Stasny, T.A.H. \& Flechtmann, C.H.W. (2003) Revised keys to world genera of Eriophyoidea (Acari: Prostigmata). Indira Publishing House, West Bloomfield, 244 pp.

Ansaloni, T. \& Perring, T.M. (2002) Biology of Aceria guerreronis Keifer on queen palms. In: XI. International Congress of Acarology, Merida, 2002. Program and Abstract Book. Universidad Nacional Autónoma de México, México, 145.

Aquino, M.L.N. \& Arruda, G.P. (1967) O agente causal da "necrose do olho do coqueiro" em Pernambuco. IPA, Recife, $33 \mathrm{pp}$.

Aquino, M.L.N., Flechtmann, C.H.W. \& Arruda, G.P. (1968) Contribuição ao estudo do ácaro causador da "necrose do olho do coqueiro" em Pernambuco e seu controle. Boletim Técnico, 34, Instituto de Pesquisa Agronômica de Pernambuco, Pernambuco, Brasil, 15pp.

Bellini, M.R., Feres, R.J.F. \& Moraes, G.J. (2005) Additional morphological characters and a new host for Aceria gymnoscuta Navia \& Flechtmann (Acari: Eriophyidae). Revista Brasileira de Zoologia, 22(2), 511-513. 
Boczek J. \& Natcheff P.D. (1989) Three new species of eriophyid mites (Acari: Eriophyoidea) from oil palms in Ivory Coast. Acarologia, 30(3), 249-253.

Briones M.L. \& Sill Jr., W.H. (1963) Habitat, gross morphology and geographical distribution of four new species of eriophyid mites from coconuts in the Philippines. FAO Plant Protection Bulletin, 11(2), 25-30.

Cabral, R.V.G. \& Carmona, M.M. (1968) Aceria guerreronis Keifer (Acarina: Eriophyidae), uma espécie nova para S. Tomé e Príncipe. Portugaliae Acta Biológica, 10(3/4), 353-358.

Cromroy, H.L. (1958) A preliminary survey of the plant mites of Puerto Rico. Journal of Agriculture of the University of Puerto Rico, 42, 39-141.

Das, A.K. \& Chakrabarti, S. (1985) Studies on eriophyid mites (Acari: Eriophyoidea) of India. XVI. One new genus and ten new species from India. Oriental Insects, 19, 133-153.

Desai, H.R., Patel, S.M., Patel,M.B. \& Saravaiya, S.N. (2003) Record of eriophyid mite, Aceria guerreronis Keifer on coconut, Cocos nucifera L. in South Gujarat. Insect Environment, 9(2), 60-61.

Doreste-S., E. (1968) El ácaro de la flor del cocotero (Aceria guerreronis Keifer) en Venezuela. Agronomia Tropical, 18(3), 370-386.

Estrada, J. \& Gonzalez, M. (1975) Daños causados en coco por Aceria guerreronis (Acarina: Eriophyidae) en Cuba. Revista de la Agricultura, 8(2), 30-34.

Fernando, L.C.P., Wickramananda, I.R., Aratchige, N.S. (2002) Status of coconut mite, Aceria guerreronis in Sri Lanka. In: Fernando, L.C.P.; Moraes, G.J.; Wickramananda, I.R. (Org.). Proceedings of the international workshop on coconut mite (Aceria guerreronis). Lunuwila: Coconut Research Institute. p.1-8

Ferreira, J.M.S., Araújo, R.P.C. \& Sarro, F.B. (2001) Mancha anelar do fruto do coqueiro: agente causal e danos. Embrapa Tabuleiros Costeiros, Aracaju, 20pp. (Documentos, 27).

Flechtmann, C.H.W. \& Santana, D.L.Q. (1997) Ocorrência de Notostrix attenuata Keifer (Acari: Eriophyidae) em coqueiros no Brasil. p.40, 16 Congresso Brasileiro de Entomologia, Sociedade Entomológica do Brasil, Salvador, Bahia, 40pp.

Flechtmann, C.H.W. (1989) Cocos weddelliana H. Wendl. (Palmae: Arecaceae), a new host plant for Eriophyes guerreronis (Keifer, 1965) (Acari: Eriophyidae) in Brazil. International Journal of Acarology, 15 (4), 241.

Flechtmann, C.H.W. (1994) Amrineus cocofolius n.g., n.sp. (Acari: Eriophyidae) from Brazil. International Journal of Acarology, 20 (1), 57-59.

Flechtmann, C.H.W. (1997) Mite (Arthropoda: Acari) associates of palms (Arecaceae) in Brazil: II. Redescription of Amrineus cocofolius Flechtmann, 1994 (Acari: Eriophyidae). International Journal of Acarology, 23(3), $195-197$.

Flechtmann, C.H.W. (1998) Mite (Arthropoda: Acari) associates of palms (Arecaceae) in Brazil. IV. Descriptions of two new species in the family Eriophyidae. International Journal of Acarology, 24 (2), 113-117.

Genty, P. \& Reyes, E. (1977) Un nouvel acarien du palmier à huile: 1’Eriophyidae Retracrus elaeis Keifer. Oléagineux, 32(6), 255-262.

Gondim Jr., M.G.C., Flechtmann, C.H.W. \& Moraes, G.J. (2000) Mite (Arthropoda: Acari) associates of palms (Arecaceae) in Brazil. IV. Descriptions of four new species in the Eriophyoidea. Systematic and Applied Acarology, 5, 99-110.

Griffith, R. (1984) The problem of the coconut mite, Eriophyes guerreronis, in the coconut groves of Trinidad \& Tobago. Proceedings of the $20^{\text {th }}$ Annual Meeting of the Caribbean Food Crops Society, Caribbean Food Crops Society, St. Croix, 128-132.

Hall, R. A., Hussey, N.W. \& Mariau, D. (1980) Results of a survey of biological control agents of the coconut mite Eriophyes guerreronis. Oléagineux, 35, 395-398.

Haq, M.A. (1999) Distribution of the coconut mite Aceria guerreronis in Peninsular India and adjacent islands. Entomon, 24(4), 371-379.

Howard, F.W., Abreu-Rodriguez, E. \& Denmark, H.A. (1990) Geographical and seasonal distribution of the coconut mite, Aceria guerreronis (Acari: Eriophyidae), in Puerto Rico and Florida, USA. Journal of Agriculture of the University of Puerto Rico, 74(3), 237-251.

Huang, K.W. (2001) The eriophyid mites of Taiwan: description of twenty-five species from Walapi. Bulletin of the National Museum of Natural Science, 13, 65-93.

Jagadiswari, R., Anand Prakash \& Sujatha, A. (2001). Incidences of the coconut mite, Aceria guerreronis Keifer (Acari: Eriophyidae) on coconut in Orissa. Journal of Applied Zoological Research, 12(2/3), 151.

Jones, D.L. (1995) Palms throughout the world. Smithsonian Institution Press, Washington D.C. Cap.1, 10-21.

Julia, J. F. \& Mariau, D. (1979) Nouvelles researches en Côte d'Ivoire sur Eriophyes guerreronis K., acarien ravageur des noix du cocotier. Oléagineux, 34, 181-189.

Kang, S.M. (1981) Malaysian eriophyid and tarsonemid mites on coconut. FAO Plant Protection Bulletin, $29(3 / 4), 79$.

Keifer, H.H. (1939a) Eriophyid studies III. Bulletin California Department of Agriculture, 28, 144-162.

Keifer, H.H. (1939b) Eriophyid studies V. Bulletin California Department of Agriculture, 28, 328-345.

Keifer, H.H. (1962a) Eriophyid studies B-6. California Department of Agriculture, Bureau of Entomology, Sacramento, $20 \mathrm{pp}$. 
Keifer, H.H. (1962b) Eriophyid studies B-7. California Department of Agriculture, Bureau of Entomology, Sacramento, $20 \mathrm{pp}$.

Keifer, H.H. (1962c) Eriophyid studies B-8. California Department of Agriculture, Bureau of Entomology, Sacramento, $20 \mathrm{pp}$.

Keifer, H.H. (1963) Eriophyid studies B-9. California Department of Agriculture, Bureau of Entomology, Sacramento, $20 \mathrm{pp}$.

Keifer, H.H. (1965) Eriophyid studies B-14. California Department of Agriculture, Bureau of Entomology, Sacramento, $20 \mathrm{pp}$.

Keifer, H.H. (1969) Eriophyid studies C-2. Agricultural Research Service, United States Department of Agriculture, Washington, 20pp.

Keifer, H.H. (1970) Eriophyid studies C-4. Agricultural Research Service, United States Department of Agriculture, Washington, 24pp.

Keifer, H.H. (1975a) Eriophyid studies C-10. Agricultural Research Service, United States Department of Agriculture, Washington, 24pp.

Keifer, H.H. (1975b) Eriophyid studies C-11. Agricultural Research Service, United States Department of Agriculture, Washington, 24pp.

Keifer, H.H. (1977) Eriophyid studies C-14. Agricultural Research Service, United States Department of Agriculture, Washington, 24pp.

Keifer, H.H. (1979) Eriophyid studies C-16. Agricultural Research Service, United States Department of Agriculture, Washington, 24pp.

Kuang, H.Y. (1991) Species and distribution of forest eriophyid mites in China. Newsletter of Pests and Diseases of Forest, 4, 37-41.

Lindquist, E.E., Sabelis, M.W. \& Bruin, J. (1996) Eriophyoid mites: their biology, natural enemies and control. Elsevier, Amsterdam, 790pp.

Lorenzi, H. (1996) Palmeiras no Brasil: exóticas e nativas. Plantarum, Nova Odessa, 303pp.

Mallik, B., Prasad, G. S., Ranganath, H.R. \& Kamali, H. (2003) Occurrence of Colomerus novahebridensis Keifer (Acari: Eriophyidae) in India. Indian Coconut Journal, 34(9): 5.

Mariau, D. (1969) Aceria guerreronis Keifer: récent ravageur de la cocoteraie Dahoméenne. Oléagineux, 24(5): 269_ 272.

Mariau, D. (1977) Aceria (Eriophyes) guerreronis: un important ravageurdes cocoteraies africaines et américaines. Oléagineux, 32, 101-108.

Meyer, M.K.P.S. (1992) African Eriophyoidea: Tumescoptes Keifer and a new genus Scoletoptus (Acari: Eriophyidae). Phytophylactica, 24(2), 131-134.

Mitrofanov, V.I., Sekerskaya, N.P. \& Sharonov, A.A. (1983) New genera and species of palm mites (Eriophyidae, Phyllocoptinae) from the Crimea [in Russian]. Zoologichskii Zhurnal, 62(1), 130-134.

Mohamed, I.I. \& El-Haidari, H. (1968) Contribution to the knowledge of the mite fauna of Iraq. Bulletin de la Societé Entomologique d'Egypte, I, 127-128.

Mohanasundaram, M. (1981) Two new species of Nalepellidae (Eriophyoidea: Acarina) from South India. Bulletin of Entomology, 22(1-2), 11-14.

Mohanasundaram, M. (1982) Four new species of Phyllocoptine mites (Eriophyidae: Acarina) from Tamil Nadu. Entomon, 7(1), 23-30, 1982.

Mohanasundaram, M. (1983) New genera and species of Eriophyoidea (Acarina) from South India. Indian Journal of Acarology, 7(2), 53-58.

Mohanasundaram, M. (1984) New eriophyid mites from India (Acarina: Eriophyoidea). Oriental Insects, 18, $251-283$.

Mohanasundaram, M. (1994) Three new species of eriophyids from Tamil Nadu (Eriophyidae: Acari). Entomon, 19(34), 95-99.

Moore, D. \& Howard, F.W. (1996) Coconuts. In: Lindquist, E.E.; Sabelis, M.W. \& J. Bruin (Org.). Eriophyoid mites: their biology, natural enemies and control. Elsevier, Amsterdam, 561-570.

Moore, D. (1986) Bract arrangement in the coconut fruit in relation to attack by the coconut mite Eriophyes guerreronis Keifer. Tropical Agriculture, 63(4), 285-288.

Moore, D., Alexander, L. \& Hall, R.A. (1989) The coconut mite, Eriophyes guerreronis Keifer in St. Lucia: yield losses and attempts to control it with acaricide, polybutene and Hirsutella fungus. Tropical Pest Management, 35, 83-89.

Navia, D. \& Flechtmann, C.H.W. (2002) Mite (Arthropoda: Acari) associates of palms (Arecaceae) in Brazil: VI. New genera and new species of Eriophyidae and Phytoptidae (Prostigmata: Eriophyoidea). International Journal of Acarology, 28 (2), 121-146.

Navia, D. \& Flechtmann, C.H.W. (2003a) Mites (Acari) of the genus Notostrix Keifer (Eriophyidae): new records, descriptions of six new species and key to the world species. Acarologia, XLIII, 271-289.

Navia, D. \& Flechtmann, C.H.W. (2003b) A new species of genus Adenoptus Mitrofanov, Sekerskaya and Sharonov (Acari: Eriophyidae) from European fan palm tree (Arecaceae) in France. International Journal of Acarology, 29(4), 
$1-4$.

Navia, D. \& Flechtmann, C.H.W. (2005) A new genus and five new species of Eriophyoidea (Prostigmata) associated with palm trees from Brazilian Amazon. Zootaxa, 1078, 41-58.

Navia, D., Moraes, G.J.de; Lofego, A.C. \& Flechtmann, C.H.W. (2005) Acarofauna associada a frutos de coqueiro (Cocos nucifera L.) de algumas localidades das Américas. Neotropical Entomology, 34(2), 349-354.

Ochoa, R., Aguilar H. \& Vargas, C. (1994) Acaros fitófagos de America Central: guia ilustrada. CATIE, Turrialba, $164 p p$.

Oldfield, G.N. (1996) Diversity and host plant specificity. In: Lindquist, E.E.; Sabelis, M.W. \& Bruin, J. (Org.). Eriophyoid mites: their biology, natural enemies and control. Elsevier, Amsterdam, 199-216.

Ortega, C.A., Rodriguez, V. \& Garibay, C.V. (1965) Investigaciones preliminares sobre el eriofido del fruto del cocotero, Aceria guerreronis Keifer, en la Costa Grande de Guerrero. Agricultura Técnica en México, 2, 222-226.

Prasad, G.S. \& Ranganath, H.R. (2000) Occurrence of nut infesting eriophyid mite Eriophyes guerreronis (K.) in coconut plantations in Andamans. Indian Coconut Journal, 31(6), 17.

Ramaraju, K. \& Rabindra, R.J. (2002) Palmyra, Borassus flabellifer L. (Palmae): a host of the coconut eriophyid mite Aceria guerreronis Keifer. Pest Management in Horticulture Ecosystems, 7(2), 149-151.

Robbs, C.F. \& Peracchi, A.L. (1965) Sobre a ocorrência de um ácaro prejudicial ao coqueiro (Cocos nucifera L.). Anais da $9^{\text {a }}$ Reunião Fitossanitária, Ministério da Agricultura, Rio de Janeiro, p.65-70.

Santana D.L.Q., Flechtmann C.H.W. \& Lima, M.F. (1994) Novos ácaros do coqueiro no Brasil. Comunicado Técnico, 3 , CPATC, EMBRAPA, Aracaju, 5pp.

Santana, D.L.Q. \& Flechtmann, C.H.W. (1998) Mite (Arthropoda: Acari) associates of palms (Arecaceae) in Brazil. I. Present status and new records. Revista Brasileira de Zoologia, 15, 959-963.

Sathiamma, B., Radhakrishnan Nair, C.P.R. \& Koshi, P.K. (1998) Outbreak of a nut infesting eriophyid mite, Eriophyes guerreronis (K.) in coconut plantations in India. Indian Coconut Journal, 29(2), 1-3.

Schliesske, J. (1988) On the gall mite fauna (Acari: Eriophyoidea) of Cocos nucifera L. in Costa Rica. Nachrichtenblatt des deutschen Pflanzenschutzdienstes, 40(8-9), 124-127.

Seguni, Z. (2000) Incidence, distribution and economic importance of the coconut eriophyid mite, Aceria guerreronis Keifer in Tanzanian coconut based cropping systems. In: $1^{\text {st }}$ International Workshop on Coconut Mite (Aceria guerreronis), Coconut Research Institute, Lunuwilla, Sri Lanka, 10.

Suarez, A. (1991) Distribución y evaluación de daños del acaro Eriophyes guerreronis en plantaciones de cocotero de la región de Baracoa. Protección de Plantas, 1(1), 75-81.

Subramanian, S. (2002) Eriophyid mite incidence on Palmyrah. Insect Environment, 8(3), 113-114.

Wei, S.G. \& Feng, Y.B. (2002) Three new species of Phyllocoptinae from China (Acari: Eriophyoidea). Acta Zootaxonomica Sinica, 27(4), 729-734.

Zuluaga, C.I. \& Sánchez, P.A. (1971) La roña o escoriación de los frutos del cocotero (Cocos nucifera L.) en Colombia. Acta Agronomica, 21(3), 133-139. 